\title{
Numerical analysis of the transpose diffusive term for viscoplastic-type non-Newtonian fluid flows using a collocated variable arrangement
}

\author{
A. Carmona ${ }^{a}$, O. Lehmkuhl ${ }^{a, b}$, C. D. Pérez-Segarra ${ }^{a}$, A. Oliva ${ }^{a}{ }^{\text {,* }}$ \\ ${ }^{a}$ Heat and Mass Transfer Technological Centre \\ Technical University of Catalonia, ETSEIAT \\ C/Colom 11, 08222 Terrassa, Spain. \\ e-mail: cttc@cttc.upc.edu,web page: http://www.cttc.upc.edu \\ ${ }^{b}$ Termo Fluids, S.L. \\ Avda. Jaquard, 97 1-E, 08222 Terrassa, Spain. \\ e-mail: termofluids@termofluids.com,web page: http://www.termofluids.com
}

${ }^{*}$ Corresponding author

Email addresses: angel@cttc.upc.edu (A. Carmona), oriol@cttc.upc.edu (O. Lehmkuhl), segarra@cttc.upc.edu (C. D. Pérez-Segarra), cttc@cttc.upc.edu (A. Oliva) 


\begin{abstract}
The aim of this work is to delve into the numerical analysis of viscoplastic-type non-Newtonian fluid flows. Specifically, improvements in the spatial discretization schemes and the temporal integration methods have been proposed to overcome the numerical problems introduced by the transpose diffusive term and associated with the velocity field discontinuity, the artificial viscous diffusion and the transpose viscous coupling. The resulting knowledge may be useful, among many other things, to improve the corresponding numerical simulations and gain insight into the underlying physics of this class of non-Newtonian fluid flows.
\end{abstract}

\title{
Nomenclature
}

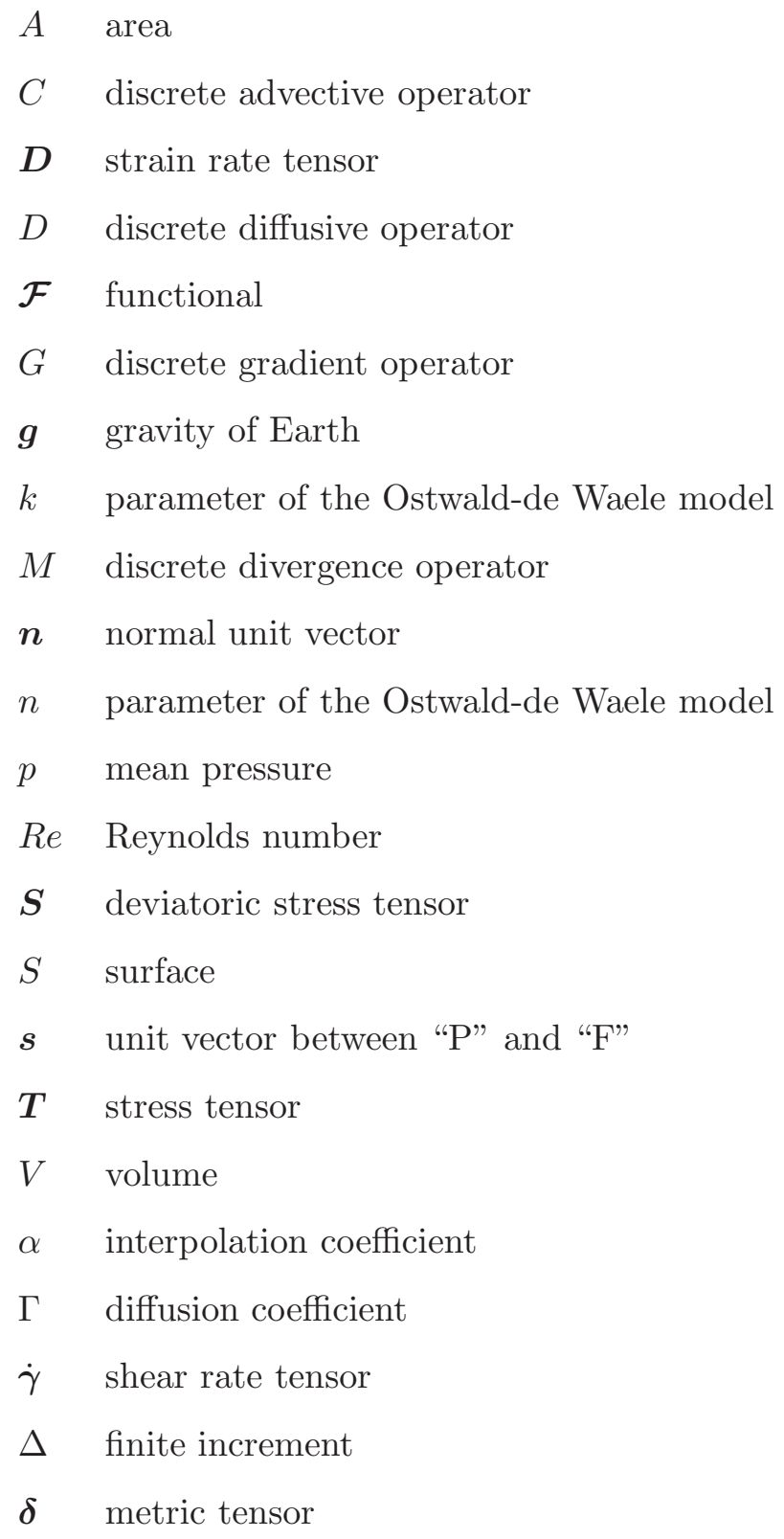




$$
\begin{array}{ll}
\eta & \text { non-Newtonian dynamic viscosity } \\
\nu & \text { momentum diffusion velocity } \\
\rho & \text { density } \\
\Omega & \text { volumetric spatial domain } \\
\partial \Omega & \text { superficial spatial domain }
\end{array}
$$

\section{Subscripts}

$$
\begin{array}{ll}
T \quad \text { transpose } \\
\sim \quad \text { pseudo-velocity }
\end{array}
$$

\section{Superscripts}

F centroid of a neighbour control volume

$f$ centroid of a control volume face

$o$ characteristic value

$P \quad$ centroid of a control volume

\section{Introduction}

CFD simulations of viscoplastic-type non-Newtonian fluid flows are of special interest due to the increment of their applications in many science and technology fields. For example, cardiovascular diseases, such as the stenosis $[1,2]$ and the aneurysms $[3,4,5]$, begin to be more often analysed by means of these computational techniques in the biomedical field. These computational techniques allow to analyse isolatedly the fluid dynamics from the other factors with which interacts in a non-linear way to jointly contribute to the disease evolution. Moreover, the corresponding numerical solutions can also complement the standard diagnoses of these diseases with a more accurate quantitative information.

Many researchers have used specific rheological laws together with the generalized Newtonian model to analyse non-Newtonian fluid flows which exhibit viscoplastic stresses (shear thinning, shear thickening and/or shear step effects). T. C. Papanastasiou [6] proposed a regularised rheological law for the Bingham plastic. Thus, the corresponding numerical results were better approximated to the experimental data. The main characteristic of this modified rheological law is its ability to deal with the sharp yield surfaces that can appear in a material, regions in which composed rheological laws would have to be applied. This type of rheological law is of interest for several reasons, including the existence of: metals which yield for strains of less than 1\%, Newtonian liquids with solid particles in suspension which show certain yield stress followed by a Newtonian behaviour or composite materials with fibres 
in suspension which exhibit some yield behaviour during their processing. B. C. Bell and K. S. Surana [7] analysed the flow of certain non-Newtonian fluids in different geometrical configurations using a power-type rheological law. Some configurations were analysed as isothermal systems, those are the parallel plates without relative motion (Hagen-Poiseuille flow), the 1:2 symmetric sudden expansion channel and the lid-driven cavity; and some other configurations were analysed as non-isothermal systems, those are the parallel plates with relative motion (Couette flow) and the 4:1 symmetric sudden contraction channel. K.A. Pericleous [8] studied the fluid flow and the heat transfer of several pseudoplastic and dilatant fluids in a differentially heated cavity using a power-type rheological law. This case is of interest because of its applications in the biotechnological field, the food industry (food processing), the metal industry (metal foundry) and in areas where the heat transfer in fine suspensions are required; among many others. E. Mitsoulis and T. Zisis [9] used the regularised Bingham rheological law [6] to analyse the corresponding flow in the lid-driven cavity. A type of non-Newtonian fluid different from that considered by B. C. Bell and K. S. Surana [7] for the same test case. R. Manica and A. L. De Bortoli [10] studied the behaviour of certain non-Newtonian fluids in a 1:3 symmetric sudden expansion channel by means of a power-type rheological law, giving special relevance to the various symmetrical and unsymmetrical solutions which appear in such flows. These expansions are relevant because of their applications in some engineering processes, e.g. cooling and heating processes, combustion processes or free jets; among many others. P. Neofytou [11] used the Quemada, Bingham (regularised) and Casson rheological laws to analyse the corresponding flow in the lid-driven cavity. The three specific rheological laws were different from that considered by B. C. Bell and K. S. Surana [7] for the same test case. M. Rudman et al. [12, 13] carried out direct numerical simulations to understand the turbulence flow of certain shear-thinning non-Newtonian fluids in a pipe. F. Zinani and S. Frey [14] analysed the fluid flow corresponding to the Casson rheological law in a 1:4 symmetric sudden expansion channel. In a latter article [15], a Carreu rheological law and a Bingham rheological law were implemented to study the corresponding fluid flows in a 4:1 symmetric sudden contraction channel and a 1:2 symmetric sudden expansion channel, respectively. L.-S. Yao and M. M. Molla $[16,17]$ proposed a regularised power-type rheological law to avoid the zero and infinite viscosity limits of the traditional power-type rheological law. Specifically, the singularities occurring on the shear-thinning or shear-thickening fluids due to the presence of the leading edge of a flat plate, where the boundary layer starts to develop, were thus eliminated. Subsequently, they studied a horizontal flat plate with uniform heat flow and forced convection [18], as well as a vertical wavy surface with a uniform temperature and natural convection [19]. M. M. Molla et al. [20] analysed the characteristics which a LES model should have in order to be conceptually consistent with the 
behaviour of certain non-Newtonian fluids. The Cross rheological law was used for this purpose. T. Ohta and M. Miyashita [21] proposed an extended subgrid scale model (Smagorinsky model) based on a spatial scale correction to predict the SGS stresses in the shear-thinning and shear-thickening non-Newtonian fluids.

Nevertheless, from our point of view, too brief numerical analyses of viscoplastic-type non-Newtonian fluid flows have been provided despite their growing presence in the field of CFD simulations. Therefore, the aim of this work is to delve into the numerical analysis of the viscoplastic-type non-Newtonian fluid flows with the objective of carrying out more advanced numerical simulations for them. This knowledge may be useful, among many other things, to gain insight into the underlying physics of this class of non-Newtonian fluid flows.

\section{Mathematical formulation}

The transport equations describing the non-Newtonian behaviour for an incompressible fluid are presented:

$$
\begin{gathered}
\nabla \cdot \boldsymbol{v}=0 \\
\rho \frac{\partial \boldsymbol{v}}{\partial t}+\rho \nabla \cdot(\boldsymbol{v} \boldsymbol{v})=-\nabla p+\nabla \cdot(\eta \nabla \boldsymbol{v})+\nabla \cdot\left(\eta(\nabla \boldsymbol{v})^{T}\right)+\rho \boldsymbol{g}
\end{gathered}
$$

The present numerical analysis is based on a finite volume discretization and a collocated variable arrangement (see Fig. 1). A structured mesh with a uniform discretization has been used in order to highlight essential numerical aspects on the test cases. However, the discrete formulation has been developed so that it can also be used on unstructured meshes. Thus, the spatial operators have been discretised $\left(\nabla \cdot \phi \approx\left(1 / \Omega_{P}\right) M_{P}, \nabla \phi \approx\left(1 / \Omega_{P}\right) G_{P}, \nabla \cdot(\Gamma \nabla \phi) \approx\left(1 / \Omega_{P}\right) D_{P}, \nabla \cdot(\rho \boldsymbol{v} \phi) \approx\left(1 / \Omega_{P}\right) C_{P}\right)$ as follows:

$$
\begin{gathered}
M_{P}=\overline{\left[\int_{\Omega} \nabla \cdot \phi d V\right]_{P}}=\sum_{f} M_{f} \phi_{f} \\
G_{P}=\overline{\left[\int_{\Omega} \nabla \phi d V\right]_{P}}=\sum_{f} G_{F} \phi_{F}-\phi_{P} \sum_{f} G_{F} \\
C_{P}=\overline{\left[\int_{\Omega} \nabla \cdot(\rho \boldsymbol{v} \phi) d V\right]_{P}}=\sum_{f} C_{F} \phi_{F}-\phi_{P} \sum_{f} C_{F}
\end{gathered}
$$




$$
D_{P}=\overline{\left[\int_{\Omega} \nabla \cdot\left(\Gamma_{\phi} \nabla \phi\right) d V\right]_{P}}=\sum_{f} D_{f} \phi_{F}-\phi_{P} \sum_{f} D_{f}
$$

Where: $M_{f}=\boldsymbol{A}_{f}, G_{F}=G_{f} \alpha_{F \rightarrow f}, G_{f}=\boldsymbol{A}_{f}=M_{f}, C_{F}=C_{f} \alpha_{F \rightarrow f}, C_{f}=\rho_{f} \boldsymbol{v}_{f} \cdot \boldsymbol{A}_{f}=\rho_{f} \boldsymbol{v}_{f} \cdot M_{f}$ and $D_{f}=\left(\Gamma_{\phi, f} A_{f}^{s}\right) / d_{P F}=\left(\Gamma_{\phi, f} A_{f}\right) /\left(d_{P F} \boldsymbol{s} \cdot \boldsymbol{n}\right)=\left(\Gamma_{\phi, f} M_{f} \cdot \boldsymbol{n}\right) /\left(d_{P F} \boldsymbol{s} \cdot \boldsymbol{n}\right)$. In addition, in order to preserve the symmetries which give rise to the conservation laws, it has been considered $\alpha_{F \rightarrow f}=0.5$ (see Ref. [22]) for the discrete representation of the mathematical problem.

Thus, the transport equations in matrix form are:

$$
\begin{gathered}
M \boldsymbol{v}_{f}=0 \\
\rho_{c} \frac{\partial \boldsymbol{v}_{c}}{\partial t}+C \boldsymbol{v}_{c}=-G p_{c}+D \boldsymbol{v}_{c}+\nabla_{h} \cdot\left(\eta\left(\nabla_{h} \boldsymbol{v}\right)^{T}\right)+B_{g}
\end{gathered}
$$

Where $c \equiv$ center and $f \equiv$ face. Furthermore $\rho_{c} \in \mathbb{R}^{3 n, 3 n}, \boldsymbol{v}_{c} \in \mathbb{R}^{3 n}, p_{c} \in \mathbb{R}^{n}, M \in \mathbb{R}^{n, m}$, $G \in \mathbb{R}^{3 n, n}, C \in \mathbb{R}^{3 n, 3 n}$ and $D \in \mathbb{R}^{3 n, 3 n}$. Here $n$ and $m$ applies for the total number of control volumes and control volume faces of the discretised spatial domain, respectively.

Moreover, the constitution equations generating the non-Newtonian behaviour are analysed (see Ref. [23]). Thus, the movement of the infinitesimal elements which form the continuous medium of a simple fluid can be described by the following kinematic variable $\boldsymbol{D}\left(X, t^{\prime}\right)$ with $-\infty<t^{\prime} \leq t$, whose choice is based on the principle of determinism, local action and invariance with respect to the reference and observation systems. However, in order to be consistent with the rheological model to be developed, a fluid without memory is assumed, which allows to disregard the determinism principle on it.

$$
\boldsymbol{T}(X, t)=\mathcal{F}[\boldsymbol{D}(X, t)]
$$

It is considered an isotropic fluid. It means that there is no prevailing direction in the physical properties. Therefore, the principal coordinate system of the strain rate tensor and the corresponding of the extra stress tensor coincide. Thereby, the previous tensorial relationship is reduced to a scalar relationship between the principal values of the tensors $\boldsymbol{T}$ and $\boldsymbol{D}$.

$$
T_{i}=\phi_{1}+\phi_{2} D_{i}+\phi_{3} D_{i}^{2}
$$


This scalar relationship must also be valid for any other coordinate system rotated with respect to the first one, due to the principle of invariance with respect to the reference system. For this reason, the above scalar relationship can be expressed as follows (theorem of representation for isotropic symmetric tensorial functions):

$$
\boldsymbol{T}=\phi_{1}\left(I_{\boldsymbol{D}}, I I_{\boldsymbol{D}}, I I I_{\boldsymbol{D}}\right) \boldsymbol{\delta}+\phi_{2}\left(I_{\boldsymbol{D}}, I I_{\boldsymbol{D}}, I I I_{\boldsymbol{D}}\right) \boldsymbol{D}+\phi_{3}\left(I_{\boldsymbol{D}}, I I_{\boldsymbol{D}}, I I I_{\boldsymbol{D}}\right) \boldsymbol{D}^{2}
$$

Where the coefficients $\phi_{1}, \phi_{2}$ and $\phi_{3}$ are only and exclusively a function of the invariants of $\boldsymbol{D}$.

$$
\begin{gathered}
I_{\boldsymbol{D}}=\operatorname{tr} \boldsymbol{D} \\
I I_{\boldsymbol{D}}=\frac{1}{2}\left[(\operatorname{tr} \boldsymbol{D})^{2}-\operatorname{tr}\left(\boldsymbol{D}^{2}\right)\right] \\
I I I_{\boldsymbol{D}}=\operatorname{det} \boldsymbol{D}
\end{gathered}
$$

The first coefficient is determined from the incompressible fluid restriction (observe that $I_{\boldsymbol{D}}=$ $\operatorname{tr} \boldsymbol{D}=0):$

$$
\phi_{1}=-p
$$

Whereas the remaining two coefficients are obtained considering a viscometric flow restriction (see Ref. [24] and observe also that $\left.I I I_{\boldsymbol{D}}=\operatorname{det} \boldsymbol{D}=0\right)$ :

$$
\eta=\frac{\phi_{2}}{2} \quad \nu_{1}=0 \quad \nu_{2}=\frac{\phi_{3}}{4}
$$

From which:

$$
\phi_{2}=2 \eta \quad \phi_{3}=4 \nu_{2}
$$

In order to continue being consistent with the rheological model which is being developed, an inelastic fluid is also assumed. This fact also avoids the unrealistic property corresponding to the coefficients $\nu_{1}=0$ and $\nu_{2} \neq 0$. Thus, the viscometric coefficient $\nu_{2}$ is considered null. 


$$
\phi_{3}=\nu_{2}=0
$$

Finally, taking also into account that $I I_{\boldsymbol{D}}=f\left(I I_{\dot{\gamma}}\right)$ and $I I_{\dot{\gamma}}=\dot{\gamma}$, the stress tensor for a viscoplastictype non-Newtonian fluid flow is:

$$
\boldsymbol{T}=-p \boldsymbol{\delta}+\boldsymbol{S} \text { where } \boldsymbol{S}=2 \eta(\dot{\gamma}) \boldsymbol{D}
$$

In the specialised literature, the non-Newtonian model which has been derived is called Generalized Newtonian Model.

\section{Numerical analysis}

In the numerical analysis of viscoplastic-type non-Newtonian fluid flows certain key aspects must be taken into account to overcome from the beginning the numerical problems introduced by the transpose diffusive term and associated with the velocity field discontinuity, the artificial viscous diffusion and the transpose viscous coupling. In this section, these problems together with some proposed solutions will be analysed.

\subsection{Spatial Discretization Schemes}

Concerning the velocity field discontinuity, the discretised transpose diffusive term should be composed of contiguous values of the collocated discrete variable, with the objective of detecting any local discontinuity for reproducing faithfully the non-Newtonian behaviour. Regarding the artificial viscous diffusion, the previous term should be cancelled under the hypothesis of incompressible fluid when the non-Newtonian viscosity takes the value of the Newtonian viscosity, this time with the objective of avoiding any non-physical diffusion for reproducing accurately the Newtonian behaviour. To face with both problems, several spatial discretization schemes have been developed and analysed for the transpose diffusive term. Previously, two identities will be used to transform the mathematical expression of this term into two equivalent expressions. The first identity is based on the divergence theorem, while the second one is based on Green's first identity. This step will be the origin of the staggered and collocated operators.

In this section, the Taylor-Green vortex (see Ref. [25]) is used as a test case in order to verify the aforementioned schemes. The specific stream function, at time $t=0$, is:

$$
\psi=\frac{1}{k^{2}} \sin \left(k x-x_{0}\right) \sin \left(k z-z_{0}\right)
$$


The analysed control volume has its centroid located at the position $x=z=3 \pi / 4$ of the region between $x_{0}=z_{0}=\pi / 2$ and $x_{f}=z_{f}=\pi$, for $k=(\pi / 2) / L$. As a previous verification for the discretised elements (velocity gradient components, shear rate and non-Newtonian viscosity) which take part in the transpose diffusion term, the approximation order of the corresponding truncation errors are verified (see Fig. 2).

\subsubsection{Staggered operator $(S O)$}

The expression for the transpose diffusive term based on the divergence theorem is:

$$
\int_{\Omega} \nabla \cdot\left(\eta(\nabla \boldsymbol{v})^{T}\right) d V=\int_{\partial \Omega}\left(\eta(\nabla \boldsymbol{v})^{T}\right) \cdot \boldsymbol{n} d S
$$

In addition, if the midpoint rule is used for the surface integral:

$$
\int_{\partial \Omega}\left(\eta(\nabla \boldsymbol{v})^{T}\right) \cdot \boldsymbol{n} d S \approx \sum_{f} \eta_{f}\left(\nabla_{h} \boldsymbol{v}\right)_{f}^{T} \cdot \boldsymbol{n} A_{f}
$$

Hence, different ways of approaching this staggered velocity gradient, as well as some of their particular characteristics, will be analysed.

Arithmetic mean (AM)

The first approach consists in an interpolation based on an arithmetic mean (see Fig. 3 for notation).

$$
\begin{aligned}
\left(\nabla_{h} \boldsymbol{v}\right)_{f} & =\frac{\left(\nabla_{h} \boldsymbol{v}\right)_{P}+\left(\nabla_{h} \boldsymbol{v}\right)_{F}}{2} \\
& =\frac{1}{2}\left[\frac{1}{\Omega_{P}} \sum_{P F} \frac{\boldsymbol{v}_{F}}{2} \boldsymbol{A}_{P F}+\frac{1}{\Omega_{F}} \sum_{F 2 F} \frac{\boldsymbol{v}_{2 F}}{2} \boldsymbol{A}_{F 2 F}\right]
\end{aligned}
$$

Thus, the spatial discretization scheme is as follows:

$$
\begin{aligned}
\nabla_{h} \cdot\left(\eta\left(\nabla_{h} \boldsymbol{v}\right)^{T}\right) & =\frac{1}{\Omega_{P}} \sum_{f} \eta_{f}\left(\nabla_{h} \boldsymbol{v}\right)_{f}^{T} \cdot \boldsymbol{A}_{f} \\
& =\frac{1}{\Omega_{P}}\left(\frac{1}{2 \Omega_{P}} \sum_{P F} \boldsymbol{A}_{P F} \frac{\boldsymbol{v}_{F}}{2}\right) \cdot \sum_{f} \eta_{f} \boldsymbol{A}_{f} \\
& +\frac{1}{\Omega_{P}} \sum_{f}\left(\frac{\eta_{f}}{2 \Omega_{F}} \sum_{F 2 F} \boldsymbol{A}_{F 2 F} \frac{\boldsymbol{v}_{2 F}}{2}\right) \cdot \boldsymbol{A}_{f}
\end{aligned}
$$


This scheme has a stencil of contiguous nodes and, from a discrete point of view, it will generate a pattern of continuous values (see Fig. 4). Therefore, the capacity of keeping velocity field discontinuities in the viscoplastic-type non-Newtonian fluid flow is eliminated by it. However, new setbacks arise from this scheme if a Newtonian fluid is considered. The first term in the Eq. 24 is cancelled while the second one does not have to do so, although the whole expression disappears in its continue version. Therefore, artificial viscous diffusion could be generated. Taking this into account and using the identity $\nabla \cdot(\nabla \boldsymbol{a})^{T}=\nabla(\nabla \cdot \boldsymbol{a})$, where $\boldsymbol{a}$ is a vector, the corresponding artificial viscous diffusion $\left(\mathrm{AVD}^{A M}\right)$ will be:

$$
\begin{aligned}
\mathrm{AVD}^{A M} & =\frac{\eta}{\Omega_{P}}\left(\frac{1}{2 \Omega_{P}} \sum_{P F} \frac{\boldsymbol{v}_{F}}{2} \cdot \boldsymbol{A}_{P F}\right) \sum_{f} \boldsymbol{A}_{f} \\
& +\frac{\eta}{\Omega_{P}} \sum_{f}\left(\frac{1}{2 \Omega_{F}} \sum_{F 2 F} \frac{\boldsymbol{v}_{2 F}}{2} \cdot \boldsymbol{A}_{F 2 F}\right) \boldsymbol{A}_{f} \\
\sum_{f} \boldsymbol{A}_{f}=0 & \frac{\eta}{\Omega_{P}} \sum_{f}\left(\frac{1}{2 \Omega_{F}} \sum_{F 2 F} \frac{\boldsymbol{v}_{2 F}}{2} \cdot \boldsymbol{A}_{F 2 F}\right) \boldsymbol{A}_{f}
\end{aligned}
$$

Fig. 5 shows the verifications which have been carried out for the transpose diffusion term that uses a spatial discretization scheme which is based on a staggered operator approximated with an arithmetic mean.

Weighted mean (WM)

In the second approach, the interpolation is based on a weighted mean.

$$
\begin{aligned}
\left(\nabla_{h} \boldsymbol{v}\right)_{f} & =\frac{\left(\nabla_{h} \boldsymbol{v}\right)_{P} \Omega_{P}+\left(\nabla_{h} \boldsymbol{v}\right)_{F} \Omega_{F}}{\Omega_{P F}} \\
& =\frac{1}{\Omega_{P F}}\left[\sum_{P F} \frac{\boldsymbol{v}_{F}}{2} \boldsymbol{A}_{P F}+\sum_{F 2 F} \frac{\boldsymbol{v}_{2 F}}{2} \boldsymbol{A}_{F 2 F}\right]
\end{aligned}
$$

Here, the spatial discretization scheme is:

$$
\begin{aligned}
\nabla_{h} \cdot\left(\eta\left(\nabla_{h} \boldsymbol{v}\right)^{T}\right) & =\frac{1}{\Omega_{P}} \sum_{f} \eta_{f}\left(\nabla_{h} \boldsymbol{v}\right)_{f}^{T} \cdot \boldsymbol{A}_{f} \\
& =\frac{1}{\Omega_{P}}\left(\frac{1}{\Omega_{P F}} \sum_{P F} \boldsymbol{A}_{P F} \frac{\boldsymbol{v}_{F}}{2}\right) \cdot \sum_{f} \eta_{f} \boldsymbol{A}_{f} \\
& +\frac{1}{\Omega_{P}} \sum_{f}\left(\frac{\eta_{f}}{\Omega_{P F}} \sum_{F 2 F} \boldsymbol{A}_{F 2 F} \frac{\boldsymbol{v}_{2 F}}{2}\right) \cdot \boldsymbol{A}_{f}
\end{aligned}
$$


Although a different type of interpolation has been done, similar observations to that of the AM can be done. Thus, if a Newtonian viscosity is considered and the identity $\nabla \cdot(\nabla \boldsymbol{a})^{T}=\nabla(\nabla \cdot \boldsymbol{a})$ is used, where $\boldsymbol{a}$ is a vector, the corresponding artificial viscous diffusion $\left(\operatorname{AVD}^{W M}\right)$ will be:

$$
\begin{aligned}
\mathrm{AVD}^{W M} & =\frac{\eta}{\Omega_{P}}\left(\sum_{P F} \frac{\boldsymbol{v}_{F}}{2} \cdot \boldsymbol{A}_{P F}\right) \sum_{f} \frac{\boldsymbol{A}_{f}}{\Omega_{P F}} \\
& +\frac{\eta}{\Omega_{P}} \sum_{f}\left(\frac{1}{\Omega_{P F}} \sum_{F 2 F} \frac{\boldsymbol{v}_{2 F}}{2} \cdot \boldsymbol{A}_{F 2 F}\right) \boldsymbol{A}_{f}
\end{aligned}
$$

This artificial viscous diffusion is only slightly different to the previous one. However, unlike the previous approach, the first term of this artificial viscous diffusion does not disappear and, therefore, also makes contribution to it. Thus, this approach could emphasize the artificial viscous diffusion with respect to that of the arithmetic mean (see Eq. 25).

Semi-direct approach $(S D)$

The last analysed approach for the staggered operator consists in composing the velocity gradient at the face from a directional velocity derivative and a transversal velocity derivative (see Fig. 6). The latter is obtained from an arithmetic mean approach for the velocity gradient at the face.

$$
\underbrace{\left(\nabla_{h} \boldsymbol{v}\right)_{f}}_{\boldsymbol{c}}=\underbrace{\frac{\boldsymbol{P F}}{|\boldsymbol{P F}|}\left(\frac{\boldsymbol{v}_{F}-\boldsymbol{v}_{P}}{|\boldsymbol{P} \boldsymbol{F}|}\right)}_{\boldsymbol{b}}+\underbrace{\left(\nabla_{h} \boldsymbol{v}\right)_{f}^{A M}-\frac{\boldsymbol{P F}}{|\boldsymbol{P F}|}\left(\frac{\boldsymbol{P F}}{|\boldsymbol{P} \boldsymbol{F}|} \cdot\left(\nabla_{h} \boldsymbol{v}\right)_{f}^{A M}\right)}_{\boldsymbol{a}}
$$

Now, the spatial discretization scheme is:

$$
\begin{aligned}
\nabla_{h} \cdot\left(\eta\left(\nabla_{h} \boldsymbol{v}\right)^{T}\right) & =\frac{1}{\Omega_{P}} \sum_{f} \eta_{f}\left(\nabla_{h} \boldsymbol{v}\right)_{f}^{T} \cdot \boldsymbol{A}_{f} \\
& =\frac{1}{\Omega_{P}} \sum_{f} \eta_{f}\left[\frac{\boldsymbol{P F}}{|\boldsymbol{P F}|}\left(\frac{\boldsymbol{v}_{F}-\boldsymbol{v}_{P}}{|\boldsymbol{P} \boldsymbol{F}|}\right)\right] \cdot \boldsymbol{A}_{f} \\
& +\frac{1}{\Omega_{P}} \sum_{f} \eta_{f}\left[\left(\nabla_{h} \boldsymbol{v}\right)_{f}^{A M, T}-\frac{\boldsymbol{P} \boldsymbol{F}}{|\boldsymbol{P F}|}\left(\frac{\boldsymbol{P F}}{|\boldsymbol{P} \boldsymbol{F}|} \cdot\left(\nabla_{h} \boldsymbol{v}\right)_{f}^{A M}\right)\right] \cdot \boldsymbol{A}_{f}
\end{aligned}
$$

For the same reason as in the SO-AM scheme, the capacity of keeping velocity field discontinuities in the viscoplastic-type non-Newtonian fluid flow is eliminated by it (see Fig. 7). However, from the point of view of a Newtonian fluid, this scheme could emphasize the artificial viscous diffusion with respect to that of the arithmetic mean (see Eq. 25). 


$$
\begin{aligned}
\mathrm{AVD}^{P L} & =\mathrm{AVD}^{A M} \\
& +\frac{\eta}{\Omega_{P}} \sum_{f}\left[\frac{\boldsymbol{P F}}{|\boldsymbol{P F}|}\left(\frac{\boldsymbol{v}_{F}-\boldsymbol{v}_{P}}{|\boldsymbol{P F}|}-\frac{\boldsymbol{P} \boldsymbol{F}}{|\boldsymbol{P F}|} \cdot\left(\nabla_{h} \boldsymbol{v}\right)_{f}^{A M}\right)\right] \cdot \boldsymbol{A}_{f}
\end{aligned}
$$

Fig. 8 shows the verifications which have been carried out for the transpose diffusion term that uses a spatial discretization scheme which is based on a staggered operator approximated with a semi-direct approach.

\subsubsection{Collocated operator ( $\mathrm{CO})$}

The expression for the transpose diffusive term based on Green's first identity is:

$$
\int_{\Omega} \nabla \cdot\left(\eta(\nabla \boldsymbol{v})^{T}\right) d V=\int_{\Omega}(\nabla \boldsymbol{v})^{T} \cdot \nabla \eta d V+\int_{\Omega} \eta \nabla \nabla \cdot \overrightarrow{\boldsymbol{v}} d V
$$

The second term becomes zero by the identity $\nabla \cdot(\nabla \boldsymbol{a})^{T}=\nabla(\nabla \cdot \boldsymbol{a})$, where $\boldsymbol{a}$ is a vector, and the continuity equation (see Eq. 2). In addition, if the midpoint rule is used to obtain an average of the transpose velocity gradient and, subsequently, the divergence theorem and the midpoint rule are used for the volume and surface integrals, respectively:

$$
\begin{aligned}
& \int_{\Omega}(\nabla \boldsymbol{v})^{T} \cdot \nabla \eta d V \approx \frac{1}{\Omega_{P}} \overline{\left[\int_{\Omega}(\nabla \boldsymbol{v})^{T} d V\right]_{P}} \cdot \int_{\Omega} \nabla \eta d V \\
& =\frac{1}{\Omega_{P}} \overline{\left[\int_{\Omega}(\nabla \boldsymbol{v})^{T} d V\right]_{P}} \cdot \int_{\partial \Omega} \eta \boldsymbol{n} d S \\
& \approx \frac{1}{\Omega_{P}} \overline{\left[\int_{\Omega}(\nabla \boldsymbol{v})^{T} d V\right]_{P}} \cdot\left(\sum_{f} \eta_{f} \boldsymbol{A}_{f}\right)
\end{aligned}
$$

Hence, the divergence theorem and the midpoint rule will be again used to approximate the collocated velocity gradient.

$$
\begin{aligned}
\nabla_{h} \cdot\left(\eta\left(\nabla_{h} \boldsymbol{v}\right)^{T}\right) & =\frac{1}{\Omega_{P}}\left[\frac{1}{\Omega_{P}} \overline{\left[\int_{\Omega}(\nabla \boldsymbol{v})^{T} d V\right]} \cdot\left(\sum_{f} \eta_{f} \boldsymbol{A}_{f}\right)\right] \\
& =\frac{1}{\Omega_{P}}\left[\left(\frac{1}{\Omega_{P}} \sum_{P F} \boldsymbol{A}_{P F} \frac{\boldsymbol{v}_{F}}{2}\right) \cdot\left(\sum_{f} \eta_{f} \boldsymbol{A}_{f}\right)\right]
\end{aligned}
$$

This scheme has a stencil of alternates nodes and, from a discrete point of view, it could keep a pattern of discontinuous values (see Fig. 9). Therefore, the capacity of keeping velocity field discontinuities in a viscoplastic-type non-Newtonian fluid flow is not eliminated by it. Moreover, 
with this scheme the symmetries which give rise to the conservation laws are preserved in a more faithful way than in the previous ones. For example, the previous discrete expression is cancelled if a Newtonian fluid is considered, as happens in its continue version. It means that there will be no artificial viscous diffusion from the beginning.

$$
\mathrm{AVD}^{C O}=\frac{1}{\Omega_{P}}\left[\left(\frac{1}{\Omega_{P}} \sum_{P F} \boldsymbol{A}_{P F} \frac{\boldsymbol{v}_{F}}{2}\right) \cdot\left(\eta \sum_{\not f} \boldsymbol{A}_{f}^{0}\right)\right]=0
$$

Fig. 10 shows the verifications which have been carried out for the transpose diffusion term with a spatial discretization scheme based on the collocated operator.

\subsubsection{Discussion}

The analysed schemes for the transpose diffusive term have allowed to highlight two of the most important problems that can appear in the numerical analysis of viscoplastic-type non-Newtonian fluid flows, the velocity field discontinuity and the artificial viscous diffusion. Specifically, the SOAM scheme is not able to keep a velocity field discontinuity (see Fig. 4), although it can introduce artificial viscous diffusion (see Eq. 25). The SO-SD scheme, from the point of view of both problems, is similar to the first one. However, its artificial viscous diffusion could even be emphasised (see Fig. 7 and Eq. 31). The CO scheme can not introduce artificial viscous diffusion (see Eq. 35), since its value is always zero. However, this scheme is able to keep a velocity field discontinuity (see Fig. 9). Briefly, up to this point, the staggered discrete operator (interpolated from the analysed collocated discrete operators) is needed to eliminate the velocity field discontinuity and reproduce faithfully the non-Newtonian behaviour, whereas the collocated discrete operator is desirable to avoid the artificial viscous diffusion and reproduce the Newtonian behaviour accurately.

Moreover, in order to overcome the numerical problems remaining in these discrete operators, a solution for each scheme is proposed. On the one hand, a unique definition for the velocity at the centroid of the control volume faces is proposed to face with the artificial viscous diffusion in the staggered discrete operator. This rationale is consistent, but it is not always numerically implemented due to the difficulties on the implicit evaluation of the transpose diffusive term. Thus, if a specific definition to face with the velocity checkerboard problem (not the pressure checkerboard problem) has been used (see Refs. [26, 27]), then this definition should be always used. Accordingly, for the SO with the AM the scheme would be: 


$$
\begin{aligned}
\nabla_{h} \cdot\left(\eta\left(\nabla_{h} \boldsymbol{v}\right)^{T}\right) & =\frac{1}{\Omega_{P}} \sum_{f} \eta_{f}\left(\nabla_{h} \boldsymbol{v}\right)_{f}^{T} \cdot \boldsymbol{A}_{f} \\
& =\frac{1}{\Omega_{P}}\left(\frac{1}{2 \Omega_{P}} \sum_{P F} \boldsymbol{A}_{P F} \boldsymbol{v}_{P F}\right) \cdot \sum_{f} \eta_{f} \boldsymbol{A}_{f} \\
& +\frac{1}{\Omega_{P}} \sum_{f}\left(\frac{\eta_{f}}{2 \Omega_{F}} \sum_{F 2 F} \boldsymbol{A}_{F 2 F} \boldsymbol{v}_{F 2 F}\right) \cdot \boldsymbol{A}_{f}
\end{aligned}
$$

And the corresponding artificial viscous diffusion:

$$
\begin{aligned}
& \mathrm{AVD}^{A M}=\frac{\eta}{\Omega_{P}}\left(\frac{1}{2 \Omega_{P}} \sum_{P F} \boldsymbol{v}_{P F} \cdot \boldsymbol{A}_{P F}\right) \sum_{\nexists} \boldsymbol{A}_{f}^{0} \\
& +\frac{\eta}{\Omega_{P}} \sum_{f}\left(\frac{1}{2 \Omega_{F}} \sum_{F 2 F} \boldsymbol{v}_{F 2 F} \cdot \boldsymbol{A}_{F 2 F} 0\right) \boldsymbol{A}_{f}=0
\end{aligned}
$$

Thereby, the artificial viscous diffusion of the SO-AM scheme would be made zero. From this point of view, the SO-SD scheme would be the only one that could generate artificial viscous diffusion. On the other hand, a specific evaluation for the non-Newtonian viscosity at the centroid of the control volume faces is proposed to deal with the velocity field discontinuity in the collocated discrete operator. Consequently, for the CO the scheme would be:

$$
\begin{aligned}
\nabla_{h} \cdot\left(\eta\left(\nabla_{h} \boldsymbol{v}\right)^{T}\right) & =\frac{1}{\Omega_{P}}\left[\frac{1}{\Omega_{P}} \overline{\left[\int_{\Omega}(\nabla \boldsymbol{v})^{T} d V\right]_{P}} \cdot\left(\sum_{f} \eta_{f} \boldsymbol{A}_{f}\right)\right] \\
& =\frac{1}{\Omega_{P}}\left[\left(\frac{1}{\Omega_{P}} \sum_{P F} \boldsymbol{A}_{P F} \frac{\boldsymbol{v}_{F}}{2}\right) \cdot\left(\sum_{P F}\left(\left(1-\alpha_{f}\right) \eta_{P}+\alpha_{f} \eta_{F}\right) \boldsymbol{A}_{P F}\right)\right] \\
\sum_{f}^{\alpha_{f}=c t e} & =\frac{1}{\boldsymbol{A}_{f}=0}\left[\left(\frac{1}{\Omega_{P}} \sum_{P F} \boldsymbol{A}_{P F} \frac{\boldsymbol{v}_{F}}{2}\right) \cdot\left(\alpha_{f} \sum_{P F} \eta_{F} \boldsymbol{A}_{P F}\right)\right]
\end{aligned}
$$

The corresponding stencil and pattern is shown in Fig. 11. It is important to be aware of the fact that the velocity at the centroid of the control volume would take part of the final scheme through $\eta_{F}$ because of its dependence on $\left(\nabla_{h} \boldsymbol{v}\right)_{F}$. If this is not fulfilled, this solution for the velocity field discontinuity may not work. From this point of view and from the numerical analysis performed up to this point, the staggered operator is considered to be a better solution for the spatial discretization of transposed diffusive term.

Regarding the truncation error of the analysed spatial discretization schemes, all of them have turned out to have an accuracy of second order in a structured mesh with a uniform discretization. 
However, the magnitude of these truncation errors varies. In the SO-AM scheme (see Fig. 5) the shear-thinning truncation error is above the reference line (the line with a $2^{\text {nd }}$ order slope will be used as a reference line in this discussion), while the shear-thickening truncation error is below the last one. In the SO-SD scheme (see Fig. 8) the magnitude of the previous errors is reversed, the shear-thinning truncation error is below the reference line while the shear-thickening truncation error is above it. It is important to note the supra-convergence properties of this scheme for a structured mesh with a uniform discretization. In the $\mathrm{CO}$ scheme (see Fig. 10) the magnitude of both truncation errors remains below the reference line.

\subsection{Temporal Integration Methods}

Concerning the transpose viscous coupling, the transpose diffusive term has been analysed in the field of the temporal integration methods in order to deal with the numerical difficulty that entail the participation of the transversal velocity components in the momentum equation through this term. Thus, the velocity-pressure coupling is analysed in order to determine which resolution method performs the temporal integration with the transposed diffusive term and the traditional diffusive term (with variable viscosity) in the most robust, efficient and accurate form. For this purpose, the basic idea behind the main classes of temporal integration methods (advanced, projected and corrected step) will be firstly exposed.

In this section, the lid-driven cavity (LDC) is used as a test case. The characteristic values have been defined from: $l_{o}=L, t_{o}=l_{o} / v_{o}, v_{o}=v_{L}, \Delta p_{o}=\rho v_{o}^{2}, \eta_{o}=k\left(v_{o} / l_{o}\right)^{n-1}$. Therefore, $L$ (cavity length) and $v_{L}$ (lid-driving velocity) are the parameters of the physical problem. Thus, the test case is completely defined by two dimensionless numbers: $\mathrm{n}$ and Re. The numerical simulations will be carried out for a Reynolds number of 100 and the numerical solutions, corresponding to a steady-state fluid flow, will be considered acceptable when the convergence error decreases to a value of $1.0 \times 10^{-11}$. For the purpose of comparing different classes of fluid, the adimensionalization of the governing equations has been carried out so that the behaviour of different types of non-Newtonian fluid flow converges to the behaviour of a certain Newtonian fluid flow in the limit case of $n \rightarrow 1^{+}$and $n \rightarrow 1^{-}$(see Fig. 12). Firstly, for this purpose, a characteristic dynamic viscosity common for all the analysed fluids has been defined from the Reynolds number and, subsequently, a consistency index specific for each one of the previous fluids has also been defined from the expression $\eta_{o}=k\left(v_{o} / l_{o}\right)^{n-1}$. Thus, the consistency index will converge to the dynamic viscosity which would have a certain Newtonian fluid $(n=1)$ in the limit case of $n \rightarrow 1^{+}$and $n \rightarrow 1^{-}$. 


\subsubsection{Advanced Step Methods}

In the temporal integration methods of advanced step (e.g. Euler [28], traditional Runge Kutta methods $[29,30])$ the temporal discretization scheme is on foreground. This means that this method is subordinated to the temporal discretization scheme and, as a consequence, in its implicit version the temporal discretization step will continue being limited by its accuracy. Thus, the temporal evolution of the physical system is totally governed by the temporal discretization scheme while the action of the temporal integration method is distributed through the temporal discretization step. As a result, these methods have highly restricted the temporal discretization step in their implicit version and, for this reason, such methods have not been analysed in this work. However, their suitability for the numerical simulation of viscoplastic-type non-Newtonian fluid flows and their analysis in future works are not ruled out. For the sake of clarity, two methods of this class are presented. The first one is the Explicit Advance Method (EAM), which here presents a first-order temporal discretization scheme:

$$
\frac{(\rho \boldsymbol{v})_{c}^{n+1}-(\rho \boldsymbol{v})_{c}^{n}}{\Delta t}=-C\left(\boldsymbol{v}_{f}^{n}\right) \boldsymbol{v}_{c}^{n}+D \boldsymbol{v}_{c}^{n}+D^{T} \boldsymbol{v}_{c}^{n}-G p_{c}^{n}
$$

In this explicit method the velocity and pressure appear uncoupled. However, its extrapolation to higher accuracy orders increases the complexity of its increment function and/or the number of evaluations of the Poisson's equation. As a result, the computational cost increases sharply. The other method is the Implicit Advance Method (IAM), which here also presents a first-order temporal discretization scheme:

$$
\frac{(\rho \boldsymbol{v})_{c}^{n+1}-(\rho \boldsymbol{v})_{c}^{n}}{\Delta t}=-C\left(\boldsymbol{v}_{f}^{n+1}\right) \boldsymbol{v}_{c}^{n+1}+D \boldsymbol{v}_{c}^{n+1}+D^{T} \boldsymbol{v}_{c}^{n+1}-G p_{c}^{n+1}
$$

In this implicit method the velocity and pressure appear coupled. In addition, as previously mentioned, the basic idea behind this method limits considerably the temporal discretization step that can be taken in order to keep the error of the temporal integration method below the error of the temporal discretization scheme.

\subsubsection{Projected Step Methods}

In the temporal integration methods of projected step (see Refs. [31, 32]) the temporal discretization scheme is on background. This means that this method should not be subordinated to the temporal discretization scheme and, as a consequence, in its implicit version the temporal discretization step will be only limited by its stability. Thus, the temporal evolution of the physical system is

partially governed by the temporal integration method through the accumulation of its error in the 
temporal discretization step and the subsequent projection of the advective velocity to satisfy continuity. The Explicit Projection Method (EPM) is the first analysed method of this class. For detailed information about the temporal discretization scheme refer to [22]:

$$
\begin{gathered}
\frac{(\rho \widetilde{\boldsymbol{v}})_{c}^{n *+\beta+\frac{1}{2}}-(\rho \boldsymbol{v})_{c}^{n+\beta-\frac{1}{2}}}{\Delta t}=-C\left(\boldsymbol{v}_{f}^{n+\beta}\right) \boldsymbol{v}_{c}^{n+\beta}+D \boldsymbol{v}_{c}^{n+\beta}+D^{T} \boldsymbol{v}_{c}^{n+\beta} \\
\frac{(\rho \boldsymbol{v})_{c}^{n+\beta+\frac{1}{2}}-(\rho \widetilde{\boldsymbol{v}})_{c}^{n *+\beta+\frac{1}{2}}}{\Delta t}=-G p_{c}^{n+\beta}
\end{gathered}
$$

$$
\frac{(\rho \boldsymbol{v})_{c}^{n+\beta+\frac{1}{2}}-(\rho \boldsymbol{v})_{c}^{n+\beta-\frac{1}{2}}}{\Delta t}=-C\left(\boldsymbol{v}_{f}^{n+\beta}\right) \boldsymbol{v}_{c}^{n+\beta}+D \boldsymbol{v}_{c}^{n+\beta}+D^{T} \boldsymbol{v}_{c}^{n+\beta}-G p_{c}^{n+\beta}
$$

In this explicit method the pressure and velocity appear uncoupled. However, unlike the EAM, the decoupling is not associated with the temporal discretization scheme, but the temporal integration method. Thus, the error of the temporal integration method is accumulated in the temporal discretization step and, subsequently, used to force continuity. A semi-explicit version (EPM2) of the previous one is:

$$
\begin{gathered}
\frac{(\rho \widetilde{\boldsymbol{v}})_{c}^{n *+\beta+\frac{1}{2}}-(\rho \boldsymbol{v})_{c}^{n+\beta-\frac{1}{2}}}{\Delta t}=-C\left(\boldsymbol{v}_{f}^{n+\beta}\right) \boldsymbol{v}_{c}^{n+\beta}+D \boldsymbol{v}_{c}^{n+\beta}+D^{T} \widetilde{\boldsymbol{v}}_{c}^{n *+\beta+\frac{1}{2}} \\
\frac{(\rho \boldsymbol{v})_{c}^{n+\beta+\frac{1}{2}}-(\rho \widetilde{\boldsymbol{v}})_{c}^{n *+\beta+\frac{1}{2}}}{\Delta t}=-G p_{c}^{n+\beta+\frac{1}{2}}
\end{gathered}
$$

$$
\frac{(\rho \boldsymbol{v})_{c}^{n+\beta+\frac{1}{2}}-(\rho \boldsymbol{v})_{c}^{n+\beta-\frac{1}{2}}}{\Delta t}=-C\left(\boldsymbol{v}_{f}^{n+\beta}\right) \boldsymbol{v}_{c}^{n+\beta}+D \boldsymbol{v}_{c}^{n+\beta}+D^{T} \widetilde{\boldsymbol{v}}_{c}^{n *+\beta+\frac{1}{2}}-G p_{c}^{n+\beta+\frac{1}{2}}
$$

It is important to be aware of the fact that in the implicit diffusive and/or advective terms (e.g. see asterisk in Eq. 46) there will be a remaining error. Another method of this class is the Implicit Projection method (IPM), which also presents a first-order temporal discretization scheme:

$$
\frac{(\rho \widetilde{\boldsymbol{v}})_{c}^{n *+1}-(\rho \boldsymbol{v})_{c}^{n}}{\Delta t}=-C\left(\widetilde{\boldsymbol{v}}_{f}^{n *+1}\right) \widetilde{\boldsymbol{v}}_{c}^{n *+1}+D \widetilde{\boldsymbol{v}}_{c}^{n *+1}+D^{T} \widetilde{\boldsymbol{v}}_{c}^{n *+1}
$$




$$
\begin{gathered}
\frac{(\rho \boldsymbol{v})_{c}^{n+1}-(\rho \widetilde{\boldsymbol{v}})_{c}^{n *+1}}{\Delta t}=-G p_{c}^{n+1} \\
\frac{(\rho \boldsymbol{v})_{c}^{n+1}-(\rho \boldsymbol{v})_{c}^{n}}{\Delta t}=-C\left(\widetilde{\boldsymbol{v}}_{f}^{n *+1}\right) \widetilde{\boldsymbol{v}}_{c}^{n *+1}+D \widetilde{\boldsymbol{v}}_{c}^{n *+1}+D^{T} \widetilde{\boldsymbol{v}}_{c}^{n *+1}-G p_{c}^{n+1}
\end{gathered}
$$

In this other implicit method the pressure and velocity appear coupled. However, unlike the IAM, the coupling is not associated with the temporal discretization scheme, but with the temporal integration method. Thus, the error of the method is accumulated in the temporal discretization step and, subsequently, used to force continuity. However, as it was previously mentioned, in the implicit advective and diffusive terms there will be a remaining error. This means that the momentum equation may need to be corrected instead of being projected, thus breaking with the philosophy of this method. To overcome this setback and ensure its correct functioning for steady-state fluid flows, a key aspect is highlighted. The error of the method must be kept below the error of the temporal discretization scheme by the end of the convergence process. Taking this into account, a new version (IPM2) of the previous one (IPM) is proposed and analysed:

$$
\begin{gathered}
\frac{(\rho \boldsymbol{v})_{c}^{n *+1}-(\rho \boldsymbol{v})_{c}^{n}}{\Delta t}=-C\left(\boldsymbol{v}_{f}^{n *+1}\right) \boldsymbol{v}_{c}^{n *+1}+D \boldsymbol{v}_{c}^{n *+1}+D^{T} \boldsymbol{v}_{c}^{n *+1}-G p_{c}^{n} \\
\frac{(\rho \widetilde{\boldsymbol{v}})_{c}^{n *+1}-(\rho \boldsymbol{v})_{c}^{n *+1}}{\Delta t}=\frac{1}{2} G p_{c}^{n} \\
\frac{(\rho \boldsymbol{v})_{c}^{n+1}-(\rho \widetilde{\boldsymbol{v}})_{c}^{n *+1}}{\Delta t}=-\frac{1}{2} G p_{c}^{n+1} \\
\frac{(\rho \boldsymbol{v})_{c}^{n+1}-(\rho \boldsymbol{v})_{c}^{n} \lim _{\Delta t}^{n * \rightarrow n}-C\left(\boldsymbol{v}_{f}^{n+1}\right) \boldsymbol{v}_{c}^{n+1}+D \boldsymbol{v}_{c}^{n+1}+D^{T} \boldsymbol{v}_{c}^{n+1}-\frac{1}{2}\left[G p_{c}^{n+1}+G p_{c}^{n}\right]}{\Delta t}
\end{gathered}
$$

Thus, whereas the original method IPM with a first-order temporal discretization scheme for the pressure term leads to the following temporal integration error:

$$
\begin{aligned}
\frac{(\rho \boldsymbol{v})_{c}^{n+1}-(\rho \widetilde{\boldsymbol{v}})_{c}^{n *+1}}{\Delta t} & =-G\left(p_{c}^{n+1}-0\right) \\
& =-\Delta t G\left(\frac{p_{c}^{n+1}-0}{\Delta t}\right)
\end{aligned}
$$


The method IPM2 with a second-order temporal discretization scheme for the pressure term provides an improved temporal integration error:

$$
\begin{aligned}
\frac{(\rho \boldsymbol{v})_{c}^{n+1}-(\rho \boldsymbol{v})_{c}^{n *+1}}{\Delta t} & =-\frac{1}{2} G\left(p_{c}^{n+1}-p_{c}^{n}\right) \\
& =-\frac{1}{2} \Delta t G\left(\frac{p_{c}^{n+1}-p_{c}^{n}}{\Delta t}\right)
\end{aligned}
$$

Briefly, the robustness and efficiency of this method during all the convergence process is improved by reducing the remaining error through the use on the momentum equation of the pressure gradient at the previous instant of time, while the accuracy of the method towards the end of the convergence process is improved by reducing its error through the use on the pressure equation of a temporal pressure derivative. Thus, the computational cost of the numerical simulations has been improved for steady-state fluid flows, especially in the case of the viscoplastic-type non-Newtonian fluids.

\subsubsection{Corrected Step Methods}

In the temporal integration methods of corrected step the temporal discretization scheme is also on background. Again, this means that this method should not be subordinated to the temporal

discretization scheme and, as a consequence, in its implicit version the temporal discretization step will be only limited by its stability. Thus, the temporal evolution of the physical system is partially governed by the temporal integration method through the accumulation of its error in the temporal discretization step and the subsequent correction of the advective velocity to satisfy continuity. One of these methods that makes use of the pressure equation in the correction step is the Implicit Corrector Method (ICM):

$$
\begin{gathered}
\frac{(\rho \boldsymbol{v})_{c}^{m *}-(\rho \boldsymbol{v})_{c}^{n}}{\Delta t}=-C\left(\boldsymbol{v}_{f}^{m *}\right) \boldsymbol{v}_{c}^{m *}+D \boldsymbol{v}_{c}^{m *}+D^{T} \boldsymbol{v}_{c}^{m *}-\frac{1}{2} G p_{c}^{m-1}-\frac{1}{2} G p_{c}^{n} \\
\frac{(\rho \widetilde{\boldsymbol{v}})_{c}^{m *}-(\rho \boldsymbol{v})_{c}^{m *}}{\Delta t}=\frac{1}{2} G p_{c}^{m-1} \\
\frac{(\rho \boldsymbol{v})_{c}^{m}-(\rho \widetilde{\boldsymbol{v}})_{c}^{m *}}{\Delta t}=-\frac{1}{2} G p_{c}^{m}
\end{gathered}
$$

$$
\frac{(\rho \boldsymbol{v})_{c}^{n+1}-(\rho \boldsymbol{v})_{c}^{n}}{\Delta t} \stackrel{\lim _{* \rightarrow m}}{=}-C\left(\boldsymbol{v}_{f}^{n+1}\right) \boldsymbol{v}_{c}^{n+1}+D \boldsymbol{v}_{c}^{n+1}+D^{T} \boldsymbol{v}_{c}^{n+1}-\frac{1}{2} G p_{c}^{n+1}-\frac{1}{2} G p_{c}^{n}
$$


In this implicit method the pressure and velocity appear coupled. However, unlike the IAM and the IPM (or the IPM2), the coupling is associated with both the temporal discretization scheme and the temporal integration method. Thus, the error of the method is accumulated in the temporal discretization step and, subsequently, used to force continuity and momentum. In this way, in the implicit advective and diffusive terms there will not be a remaining error (observe the absence of asterisk in Eqs. 59 and 62). A new version (ICM2) of the previous one (ICM), that makes use of the pressure correction equation instead of the pressure equation, is presented:

$$
\begin{gathered}
\frac{(\rho \boldsymbol{v})_{c}^{m *}-(\rho \boldsymbol{v})_{c}^{n}}{\Delta t}=-C\left(\boldsymbol{v}_{f}^{m *}\right) \boldsymbol{v}_{c}^{m *}+D \boldsymbol{v}_{c}^{m *}+D^{T} \boldsymbol{v}_{c}^{m *}-G p_{c}^{m-1} \\
\frac{(\rho \boldsymbol{v})_{c}^{m}-(\rho \boldsymbol{v})_{c}^{m *}}{\Delta t}=-C\left(\boldsymbol{v}_{f}^{\prime}\right) \boldsymbol{v}_{c}^{\prime}+D \boldsymbol{v}_{c}^{\prime}+D^{T} \boldsymbol{v}_{c}^{\prime}-G p_{c}^{\prime} \\
\frac{(\rho \boldsymbol{v})_{c}^{n+1}-(\rho \boldsymbol{v})_{c}^{n}}{\Delta t} \stackrel{\lim _{m * m}^{m *}}{=}-C\left(\boldsymbol{v}_{f}^{n+1}\right) \boldsymbol{v}_{c}^{n+1}+D \boldsymbol{v}_{c}^{n+1}+D^{T} \boldsymbol{v}_{c}^{n+1}-G p_{c}^{n+1}
\end{gathered}
$$

The form in which this new version is closed can give rise to the SIMPLE [33] or SIMPLEC [34] algorithm, among many others.

\subsubsection{Discussion}

For steady-state fluid flows (see Figs. 13(a)-13(c)), the explicit projection method (EPM) has turned out to be robust and accurate, although its efficiency is far from the optimum. The semi-explicit projection method (EPM2) behaves similarly to the EPM. However, this method is considered not as robust as the previous one because of its convergence problems. For example, for the dilatant fluid with the mesh of $161 \times 1 \times 161 \mathrm{CVs}$ the EPM2 divergence. On the other hand, the implicit correction method (ICM) shows initially a good efficiency, although the efficiency finally move away from the optimum due to the deterioration of the convergence ratio between ranging values from 0.5 to 0.01 . Finally, the implicit projection method with a special treatment for the pressure (IPM2) has turned out to be robust and accurate, while it presents an efficiency that appears to be much closer to the optimum than in the other analysed methods. As can be observed in the aforementioned figures, the last fact is specially relevant for the viscoplastic-type non-Newtonian fluids.

Regarding the numerical solutions, the method EPM provides numerical solutions (see Fig. 14(a)) in good agreement with those by Guia et al. [35] for the Newtonian fluid and with those by Bell and Surana [7] for the analysed non-Newtonian fluids. However, the method EPM2 has not been able to 
provide a converged numerical solution for the shear-thickening fluid and the mesh of 161x161 CVs (see Fig. 14(b)). On the contrary, the methods IPM2 and ICM provide again numerical solutions (see Figs. 14(d) and 14(c)) in good agreement with those by Guia et al. [35] for the Newtonian fluid and with those by Bell and Surana [7] for the analysed non-Newtonian fluids.

\section{Conclusions}

This work has delved into the numerical analysis of viscoplastic-type non-Newtonian fluid flows, specifically in the spatial discretization schemes and the temporal integration methods, to overcome the numerical problems introduced by the transpose diffusive term and associated with the velocity field discontinuity, the artificial viscous diffusion and the transpose viscous coupling.

Firstly, some spatial discretization schemes have been developed and analysed to face with the velocity field discontinuity and the artificial viscous diffusion. On the one hand, the numerical problem associated with the velocity field discontinuity is due to a collocated discrete operator which is based on alternate values of the collocated discrete variable. Specifically, the transpose diffusive term based on this operator will be able to keep velocity field discontinuities, whereas the one based on a staggered discrete operator will not keep them. On the other hand, the numerical problem associated with the artificial viscous diffusion is due to a staggered discrete operator which is based on spatial interpolations of the previous collocated discrete operators. Concretely, the transpose diffusive term based on this operator will be able to introduce artificial viscous diffusion, whereas the one based on a collocated discrete operator will not introduce it. However, the source of both problems is the initial absence of the variable at the center of the control volume in the original collocated discrete operators. Moreover, in order to overcome the numerical problems remaining in these discrete operators, a solution for each scheme has been proposed in this work. A specific evaluation for the non-Newtonian viscosity at the control face in order to avoid the velocity field discontinuity in the collocated discrete operator and a unique definition for the velocity at the control face in order to eliminate the artificial viscous diffusion in the staggered discrete operator.

Secondly, some temporal integration methods have been used and analysed to deal with the transpose viscous coupling. The numerical problem associated with the transpose viscous coupling is due to the contribution of the transversal velocity components in the momentum equation through the transpose diffusive term, which is an inherent problem of this equation. Thus, the velocity-pressure coupling has been analysed in order to determine which resolution method performs the temporal integration with the transposed diffusive term and the traditional diffusive term (with variable viscosity) in the most robust, efficient and accurate form. Through this analysis, it has been possible to 
observe how the implicit methods based on a projector step with a special treatment for the pressure term (IPM2) are especially more competitive than the other ones for the steady-state fluid flow of viscoplastic-type non-Newtonian fluids. The key aspect of its high performance has turn out to be the temporal treatment of the pressure term.

Finally, it is expected that the present numerical analysis also contributes to future works in which the turbulent flow of viscoplastic-type non-Newtonian fluids is involved.

\section{Acknowledgments}

This work has been financially supported by the Spanish Ministerio de Ciencia e Innovación (Ref. ENE2010-17801).

\section{References}

[1] M. Rosenfeld, "Validation of numerical simulation of incompressible pulsatile flow in a constricted channel," Computers and Fluids, vol. 22, pp. 139-156, 1993.

[2] M. Rosenfeld, "A numerical study of pulsating flow behind a constriction," J. of Fluid Mechanics, vol. 30, pp. 203-223, 1995.

[3] S. C. M. Yu, W. K. Chan, B. T. H. Ng, and L. P. Chua, "A numerical investigation on the steady and pulsatile flow characteristics in axi-symmetric abdominal aortic aneurysm models with some experimental evaluation," J. of Medical Engineering 6 Technology, vol. 23, pp. 228-239, 1999.

[4] S. C. M. Yu, "Steady and pulsatile flow studies in abdominal aortic aneurysm models using particle image velocimetry," Int. J. of Heat and Fluid Flow, vol. 21, pp. 74-83, 2000.

[5] T. Yip and S. Yu, "Oscillatory flows in straight tubes with an axisymmetric bulge," Experimental Thermal and Fluid Science, vol. 26, pp. 947-961, 2002.

[6] T. C. Papanastasiou, "Flows of Materials with Yiels," J. of Rheology, vol. 31, pp. 385-404, 1987.

[7] B. Bell and K. Surana, "p-version least squares finite element formulation for two-dimensional, incompressible, non-Newtonian isothermal and non-isothermal fluid flow," Int. J. for Numerical Methods in Fluids, vol. 18, pp. 127-162, 1994.

[8] K. Pericleous, "Heat transfer in differentially heated non-Newtonian cavities," Int. J. of Numerical Methods for Heat \& Fluid Flow, vol. 4, pp. 229-248, 1994. 
[9] E. Mitsoulis and T. Zisis, "Flow of Bingham plastics in a lid-driven square cavity," J. NonNewtonian Fluid Mech., vol. 101, pp. 173-180, 2001.

[10] R. Manica and A. D. Bortoli, "Simulation of sudden expansion flows for power-law fluids," J. of Non-Newtonian Fluid Mechanics, vol. 121, pp. 35-40, 2004.

[11] P. Neofytou, "A 3rd order upwind finite volume method for generalised Newtonian fluid flows," Advances in Engineering Software, vol. 36, no. 10, pp. 664-680, 2005.

[12] M. Rudman, H. Blackburn, L. Graham, and L. Pullum, "Turbulent pipe flow of shear-thinning fluids," J. Non-Newtonian Fluid Mechanics, vol. 118, pp. 33-48, 2004.

[13] H. B. M. Rudman, "Direct numerical simulation of turbulent non-Newtonian flow using a spectral element method," Applied Mathematical Modelling, vol. 30, pp. 1229-1248, 2006.

[14] F. Zinani and S. Frey, "Galerkin least-squares finite element approximations for isochoric flows of viscoplastic liquids," J. of Fluids Engineering, vol. 128, pp. 856-863, 2005.

[15] F. Zinani and S. Frey, "Galerkin least-squares solutions for purely viscous flows of shear-thinning fluids and regularized yield stress fluids," J. of the Brazilian Society of Mechanical Sciences and Engineering, vol. 29, pp. 432-443, 2007.

[16] L.-S. Yao and M. Molla, "Non-Newtonian fluid flow on a flat plate part 1: boundary layer," J. of Thermophysics and Heat Transfer, vol. 22, no. 4, pp. 758-761, 2008.

[17] L.-S. Yao and M. Molla, "Non-Newtonian fluid flow on a flat plate part 2: heat transfer," J. of Thermophysics and Heat Transfer, vol. 22, no. 4, pp. 762-765, 2008.

[18] M. M. Molla and L. S. Yao, "The flow of non-Newtonian fluids on a flat plate with a uniform heat flux," J. of Heat Transfer, vol. 131, no. 1, p. 011702, 2009.

[19] M. M. Molla and L. S. Yao, "Non-Newtonian natural convection along a vertical heated wavy surface using a modified power-Law viscosity model," J. of Heat Transfer, vol. 131, no. 1, p. 012501, 2009 .

[20] M. M. Molla, A. Hossain, B.-C. Wang, and D. C. S. Kuhn, "Large-eddy simulation of pulsatile non-newtonian flow in a constricted channel," Progress in Computational Fluid Dynamics, vol. 12, pp. 231-242, 2012. 
[21] T. Ohta and M. Miyashita, "DNS and LES with an extended Smagorinsky model for wall turbulence in non-Newtonian viscous fluids," J. of Non-Newtonian Fluid Mechanics, vol. 206, no. 0, pp. 29-39, 2014.

[22] R. W. C. P. Verstappen and A. E. P. Veldman, "Symmetry-preserving discretization of turbulent flow," J. of Computational Physics, vol. 187, no. 1, pp. 343-368, 2003.

[23] J. G. Oldroyd, "On the formulation of rheological equations of state," Proceedings of the Royal Society of London. Series A. Mathematical and Physical Sciences, vol. 200, no. 1063, pp. 523-541, 1950.

[24] A. M. Robertson, "Review of relevant continuum mechanics," Hemodynamical Flows: Modeling, Analysis and Simulation, vol. 37, pp. 1-62, 2008.

[25] G. I. Taylor and A. E. Green, "Mechanism of the production of small eddies from large ones," Proceedings of the Royal Society of London. Series A, Mathematical and Physical Sciences, vol. 158, no. 895, pp. 499-521, 1937.

[26] S. Rubin, "Numerical studies of incompressible viscous flow in a driven cavity," $N A S A-S P$-378, 1975 .

[27] C. Rhie and W. Chow, "Numerical study of the turbulent flow past an airfoil with trailing edge separation," AIAA Journal, vol. 21, pp. 1525-1532, 1983.

[28] L. Euler, Institutionum calculi integralis. Petropoli: Impensis Academiae Imperialis Scientiarum, 1768-70.

[29] C. Runge, "Über die numerische auflösung von differentialgleichungen," Math. Ann., vol. 46, pp. 167-178, 1895.

[30] M. W. Kutta, "Beitrag zur näherungsweisen integration totaler differentialgleichungen," Z. Math. Phys., vol. 46, pp. 435-453, 1901.

[31] R. Temam, "Une méthode d'approximation de la solution des équations de Navier-Stokes," Bull. Soc. Math. France, vol. 96, pp. 115-152, 1968.

[32] A. Chorin, "A numerical method for solving incompressible viscous flow problems," Journal of Computational Physics, vol. 2, no. 1, pp. 12-26, 1967. 
[33] S. Patankar and D. Spalding, "A calculation procedure for heat, mass and momentum transfer in three-dimensional parabolic flows," Int. J. of Heat and Mass Transfer, vol. 15, no. 10, pp. 1787 1806, 1972.

[34] J. P. Van Doormaal and G. D. Raithby, "Enhancements of the simple method for predicting incompressible fluid flows," Numerical Heat Transfer, vol. 7, no. 2, pp. 147-163, 1984.

[35] U. Ghia, K. Ghia, and C. Shin, "High-Re solutions for incompressible flow using the Navier-Stokes equations and a multigrid method," J. of Computational Physics, vol. 48, pp. 387-411, 1982. 


\section{List of Figures}

Figure 1: Control volume $\mathrm{P}$ and contiguous neighbours $\mathrm{F}$

Figure 2: Truncation error of the velocity gradient components, the shear rate and the non-Newtonian viscosity. The divergence theorem for the volume integrals and the midpoint rule for the surface integrals have been used

Figure 3: Control volume P, contiguous neighbours F and neighbours's neighbours $2 \mathrm{~F}$

Figure 4: Stencil and pattern for the SO scheme and the AM approach

Figure 5: Truncation error of the transpose diffusive term for the shear-thinning and shear-thickening fluids using the SO scheme and the AM approach

Figure 6: Face of a control volume where the velocity gradient (c) is composed from a directional velocity derivative (b) and a transversal velocity derivative (a) for the approach SD

Figure 7: Stencil and pattern for the SO scheme and the SD approach

Figure 8: Truncation error of the transpose diffusive term for the shear-thinning and shear-thickening fluids using the SO scheme and the SD approach

Figure 9: Stencil and pattern for the CO scheme

Figure 10: Truncation error of the transpose diffusive term for the shear-thinning and shear-thickening fluids using the CO scheme

Figure 11: Stencil and pattern for the CO scheme and a interpolation for $\eta_{f}$ depending on $\boldsymbol{v}_{P}$

Figure 12: Consistency index of certain pseudoplastic and dilatant fluids approaching to a Newtonian behavior in the limit case of $n \rightarrow 1^{+}$and $n \rightarrow 1^{-}$

Figure 13: Convergence history of several temporal integration methods (runtime of $\sim 3$ days, the final convergence error will be of $\left.1.0 \times 10^{-11}\right)$. Where EPM is the explicit projection method, EPM2 is a semi-explicit version of the EPM, IPM2 is an improved version of the implicit projection method (IPM) and ICM is the implicit corrector method

Figure 14: W-Velocity profiles for several temporal integration methods (final convergence error of $\left.1.0 \times 10^{-11}\right)$. Where EPM is the explicit projection method, EPM2 is a semi-explicit version of the EPM, IPM2 is an improved version of the implicit projection method (IPM) and ICM is the implicit corrector method 


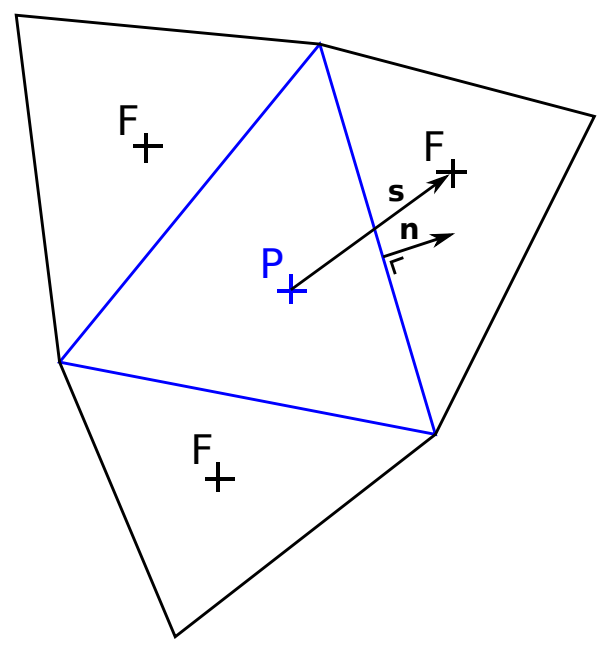

Figure 1: Control volume $\mathrm{P}$ and contiguous neighbours $\mathrm{F}$ 


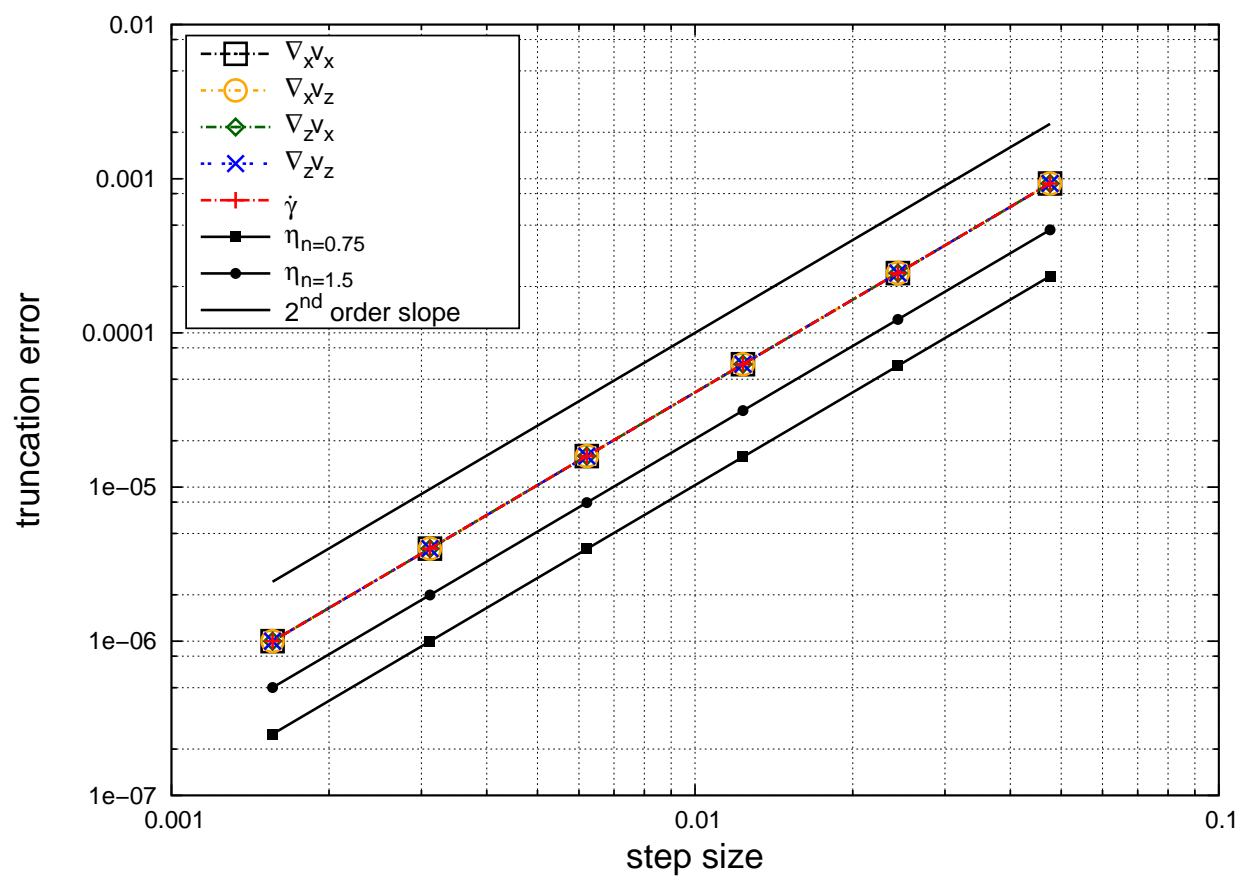

Figure 2: Truncation error of the velocity gradient components, the shear rate and the non-Newtonian viscosity. The divergence theorem for the volume integrals and the midpoint rule for the surface integrals have been used 


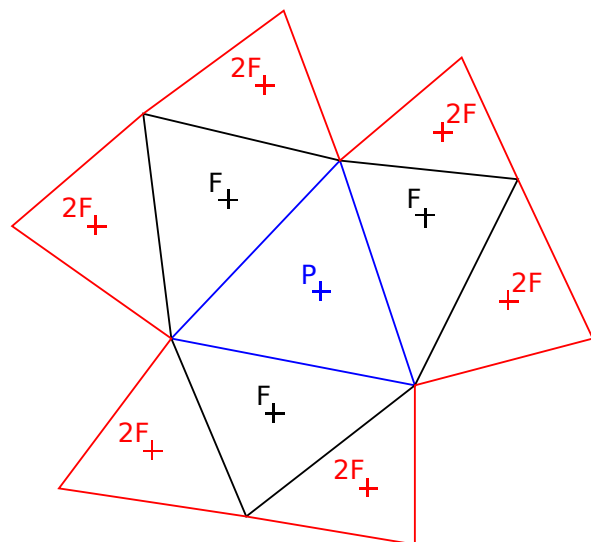

Figure 3: Control volume P, contiguous neighbours F and neighbours's neighbours $2 \mathrm{~F}$ 


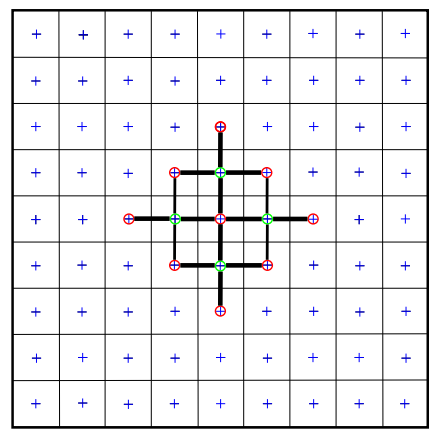

Figure 4: Stencil and pattern for the SO scheme and the AM approach 


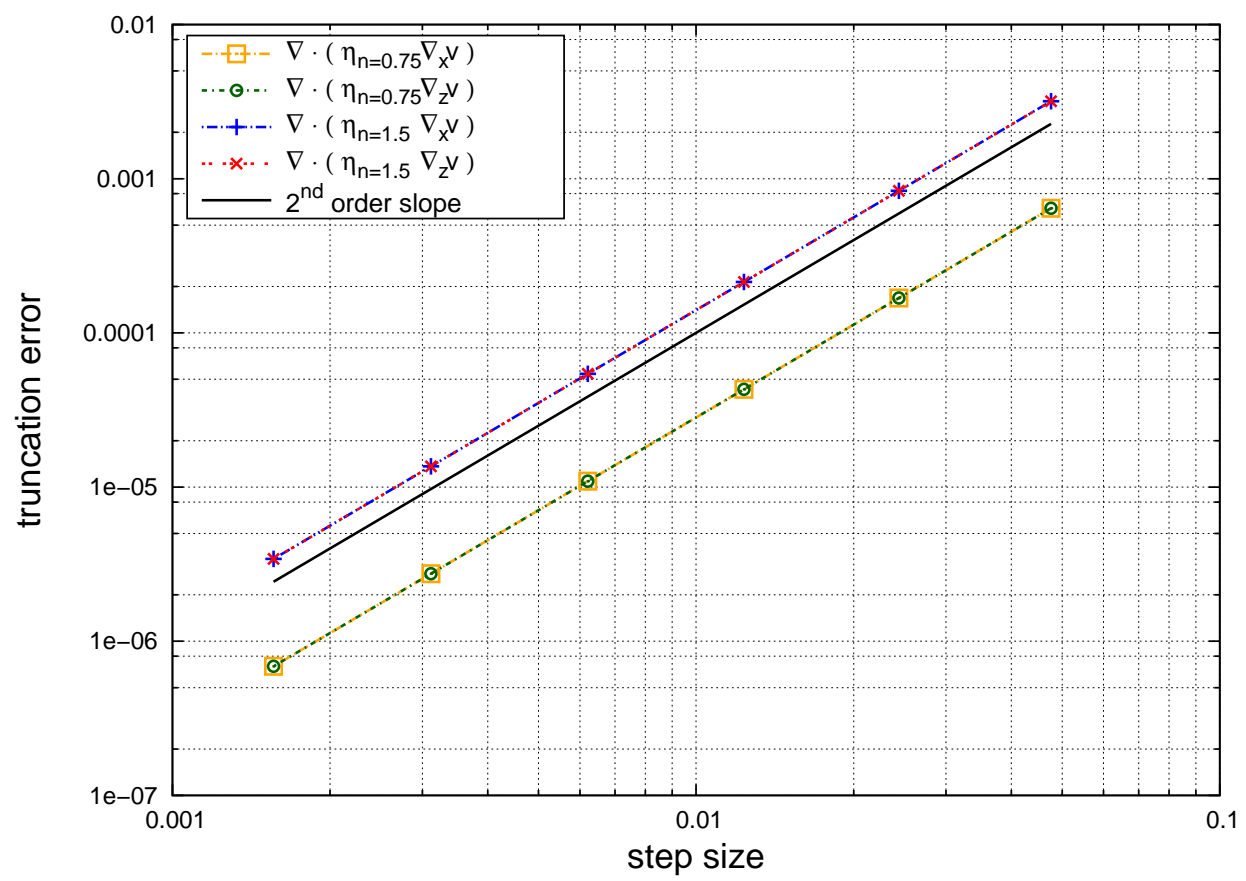

Figure 5: Truncation error of the transpose diffusive term for the shear-thinning and shear-thickening fluids using the SO scheme and the AM approach 


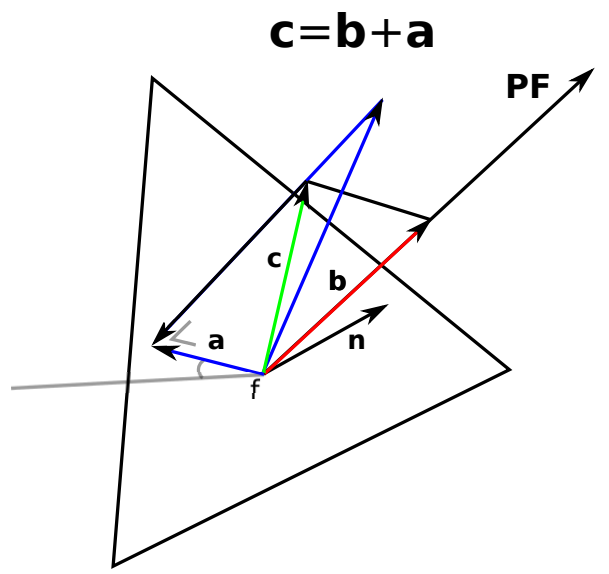

Figure 6: Face of a control volume where the velocity gradient (c) is composed from a directional velocity derivative (b) and a transversal velocity derivative (a) for the approach SD 


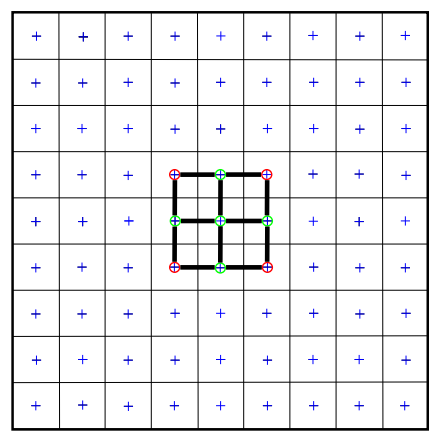

Figure 7: Stencil and pattern for the SO scheme and the SD approach 


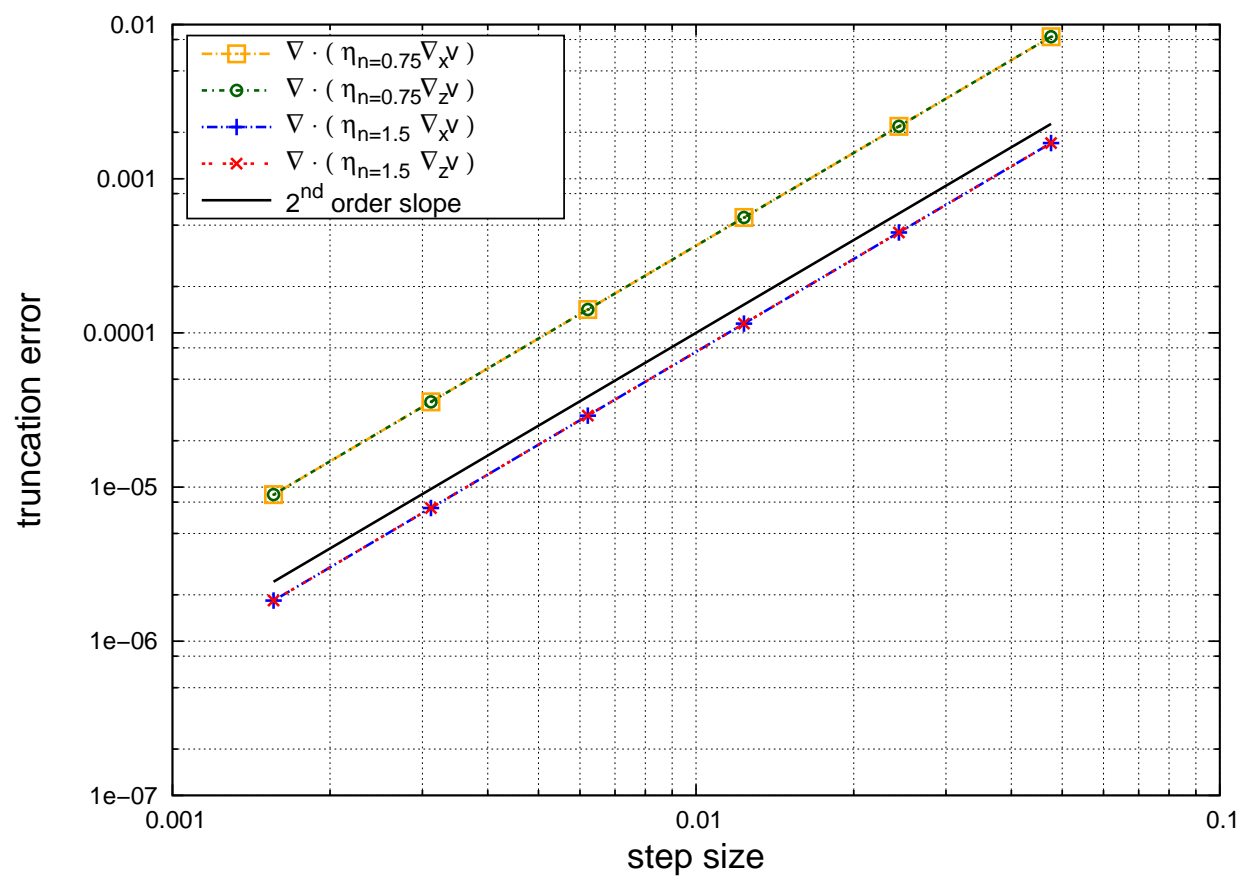

Figure 8: Truncation error of the transpose diffusive term for the shear-thinning and shear-thickening fluids using the SO scheme and the SD approach 


\begin{tabular}{|c|c|c|c|c|c|c|c|c|}
\hline+ & $\bullet$ & + & $\bullet$ & + & $\bullet$ & + & $\bullet$ & + \\
\hline$\bullet$ & + & $\bullet$ & + & $\bullet$ & + & $\bullet$ & + & $\bullet$ \\
\hline+ & $\bullet$ & + & $\bullet$ & + & $\bullet$ & + & $\bullet$ & + \\
\hline$\bullet$ & + & $\bullet$ & + & $\bullet$ & + & $\bullet$ & + & $\bullet$ \\
\hline+ & $\bullet$ & + & $\bullet$ & & $\bullet$ & + & $\bullet$ & + \\
\hline$\bullet$ & + & $\bullet$ & + & $\bullet$ & + & $\bullet$ & + & $\bullet$ \\
\hline+ & $\bullet$ & + & $\bullet$ & + & $\bullet$ & + & $\bullet$ & + \\
\hline$\bullet$ & + & $\bullet$ & + & $\bullet$ & + & $\bullet$ & + & $\bullet$ \\
\hline+ & $\bullet$ & + & $\bullet$ & + & $\bullet$ & + & $\bullet$ & + \\
\hline
\end{tabular}

Figure 9: Stencil and pattern for the CO scheme 


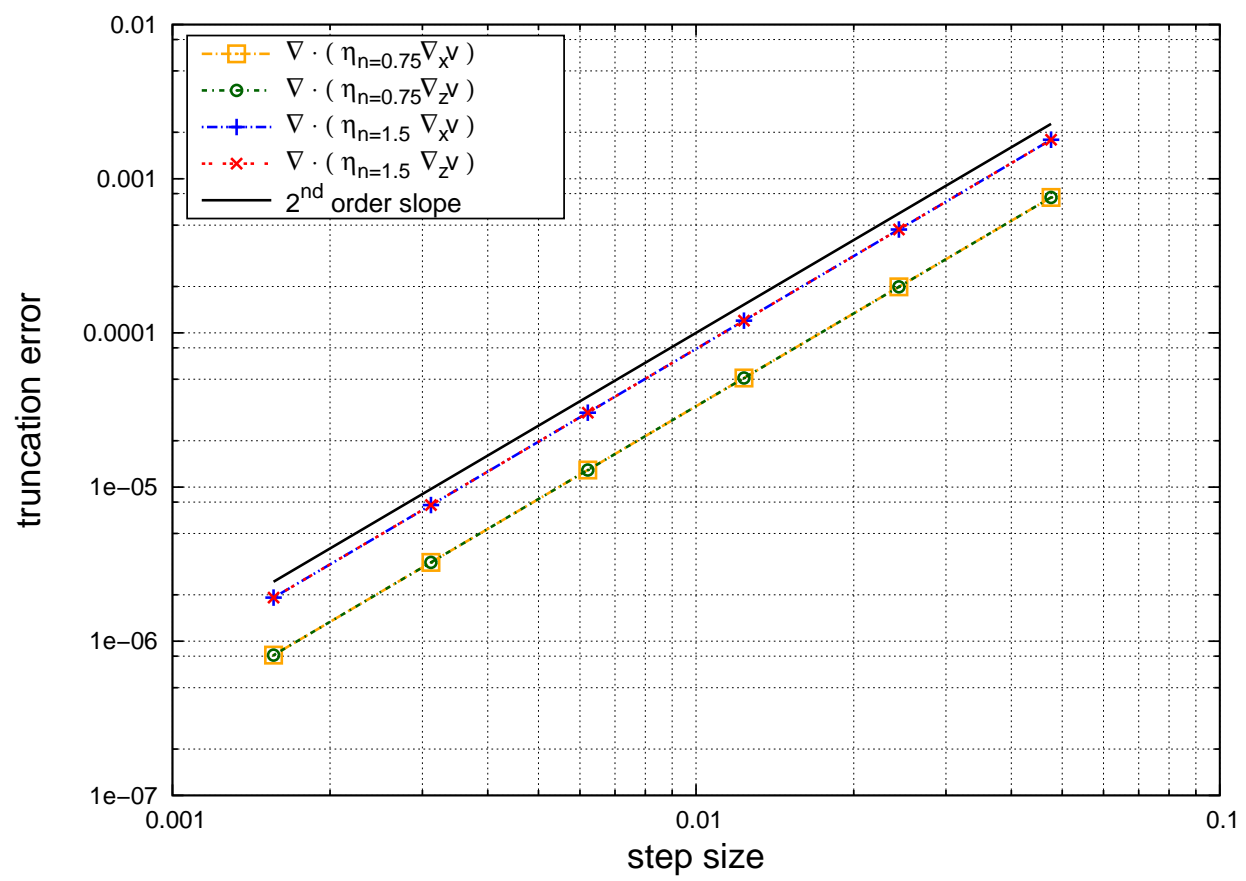

Figure 10: Truncation error of the transpose diffusive term for the shear-thinning and shear-thickening fluids using the CO scheme 


\begin{tabular}{|c|c|c|c|c|c|c|c|c|}
\hline+ & + & + & + & + & + & + & + & + \\
\hline+ & + & + & + & + & + & + & + & + \\
\hline+ & + & + & + & + & + & + & + & + \\
\hline+ & + & + & + & $\oplus$ & + & + & + & + \\
\hline+ & + & + & $\oplus-$ & $-\phi$ & $-\oplus$ & + & + & + \\
\hline+ & + & + & + & $d$ & + & + & + & + \\
\hline+ & + & + & + & + & + & + & + & + \\
\hline+ & + & + & + & + & + & + & + & + \\
\hline+ & + & + & + & + & + & + & + & + \\
\hline
\end{tabular}

Figure 11: Stencil and pattern for the CO scheme and a interpolation for $\eta_{f}$ depending on $\boldsymbol{v}_{P}$ 


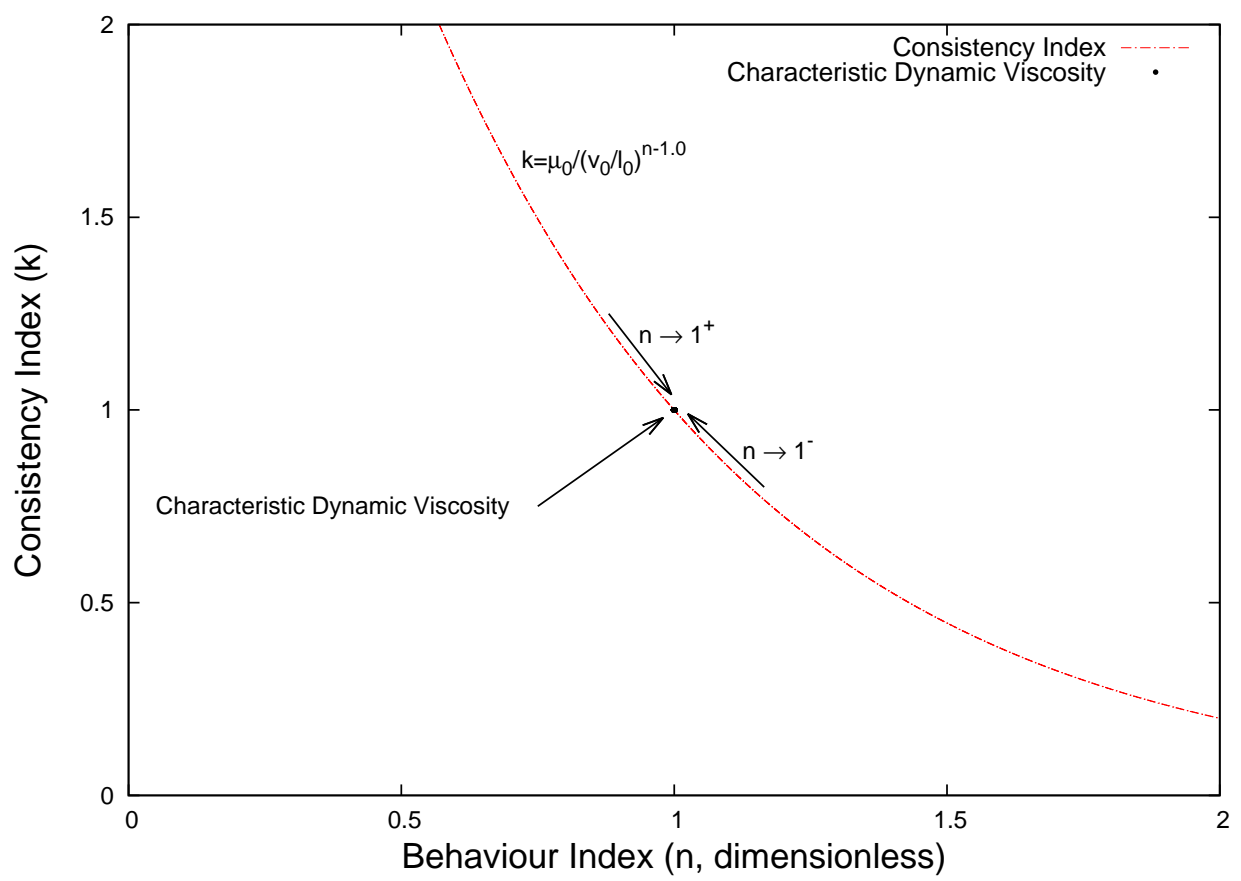

Figure 12: Consistency index of certain pseudoplastic and dilatant fluids approaching to a Newtonian behavior in the limit case of $n \rightarrow 1^{+}$and $n \rightarrow 1^{-}$ 


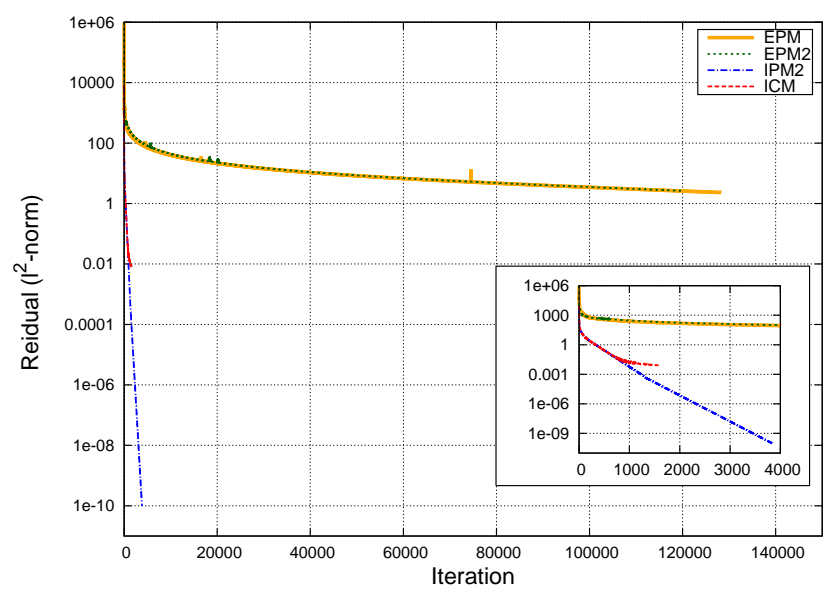

(a) Pseudoplactic fluid $(\mathrm{n}=0.75)$

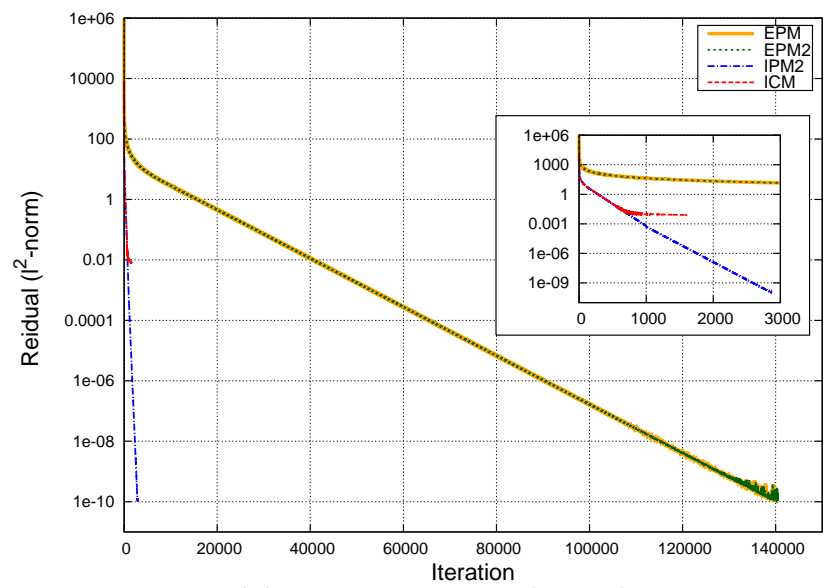

(b) Newtonian fluid $(\mathrm{n}=1.0)$

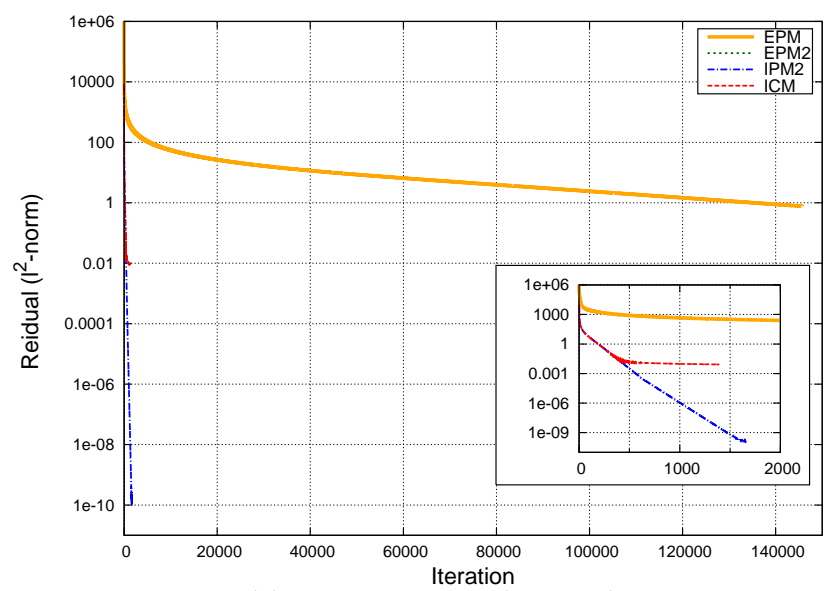

(c) Dilatant fluid $(\mathrm{n}=1.50)$

Figure 13: Convergence history of several temporal integration methods (runtime of $\sim 3$ days, the final convergence error will be of $1.0 \times 10^{-11}$ ). Where EPM is the explicit projection method, EPM2 is a semi-explicit version of the EPM, IPM2 is an improved version of the implicit projection method (IPM) and ICM is the implicit corrector method 


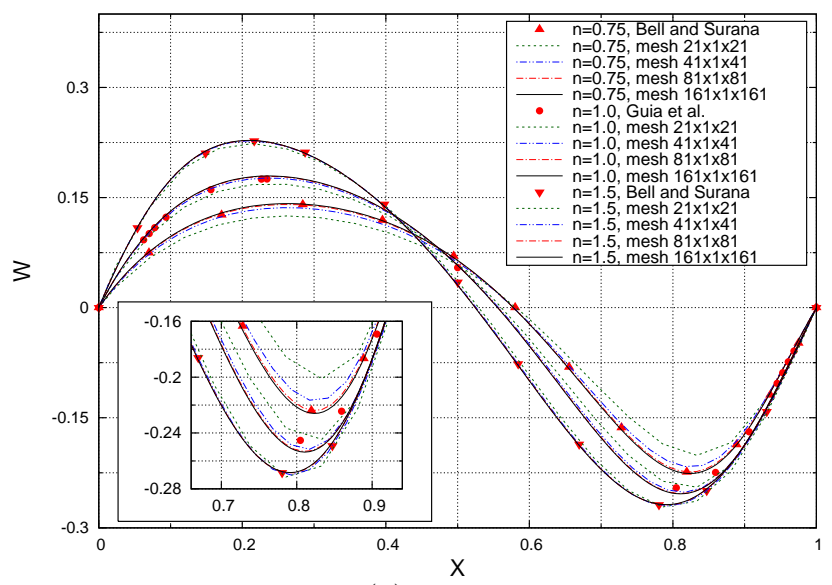

(a) EPM

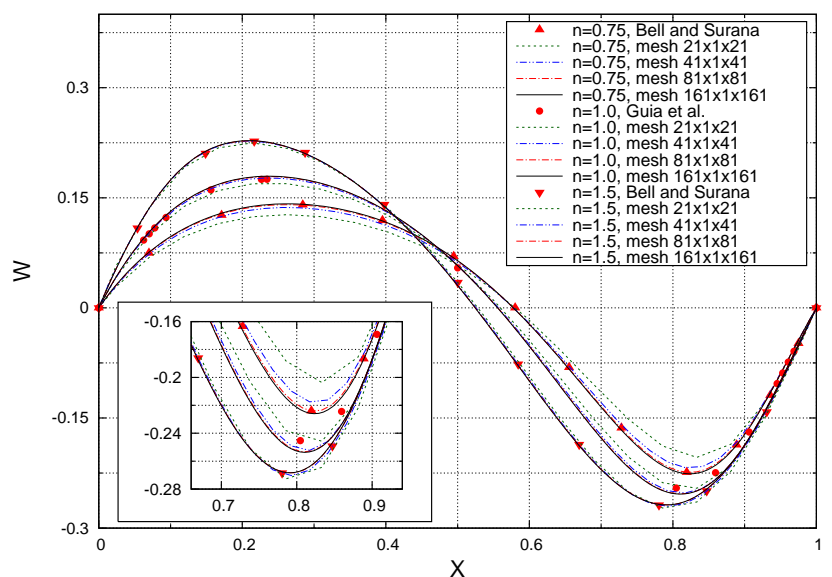

(c) IPM2

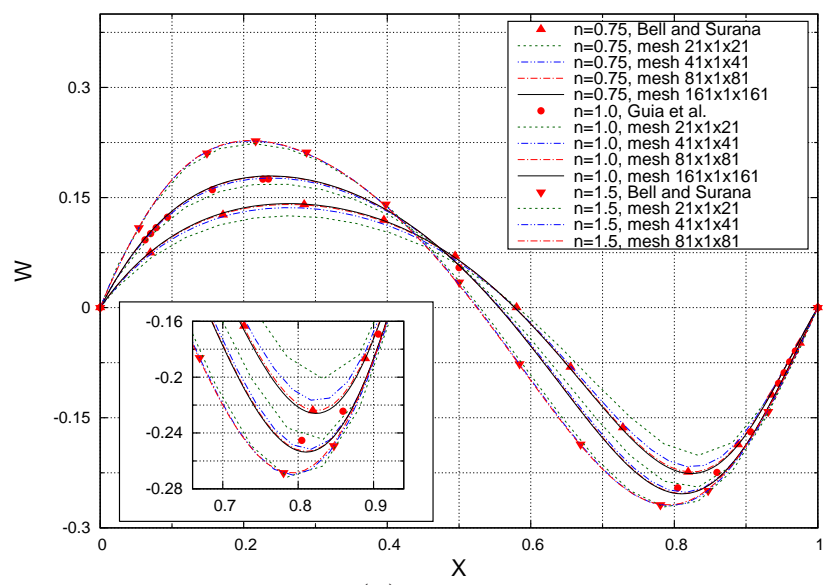

(b) EPM2

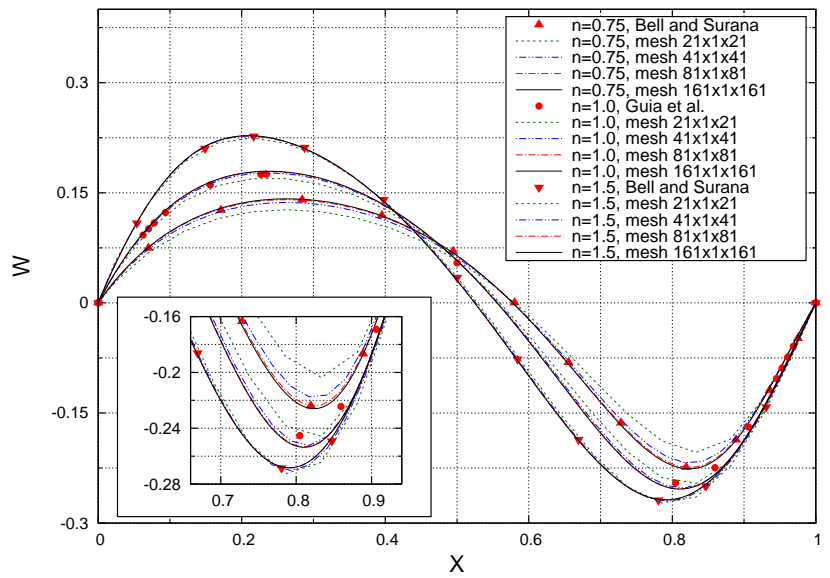

(d) ICM

Figure 14: W-Velocity profiles for several temporal integration methods (final convergence error of $1.0 \times 10^{-11}$ ). Where EPM is the explicit projection method, EPM2 is a semi-explicit version of the EPM, IPM2 is an improved version of the implicit projection method (IPM) and ICM is the implicit corrector method 